\title{
BeppoSAX/PDS serendipitous detections at high galactic latitudes
}

\author{
R. Landi ${ }^{1,2}$, A. Malizia ${ }^{1}$, and L. Bassani ${ }^{1}$ \\ 1 INAF - Istituto di Astrofisica Spaziale e Fisica Cosmica, Sezione di Bologna, via Gobetti 101, 40129 Bologna, Italy \\ e-mail: landi@bo.iasf.cnr.it \\ 2 Dipartimento di Fisica, Università degli Studi di Bologna, Viale C. Berti Pichat 6/2, 40127 Bologna, Italy
}

Received 28 July 2004 / Accepted 7 June 2005

\begin{abstract}
At a flux limit of $\sim 10^{-11} \mathrm{erg} \mathrm{cm}^{-2} \mathrm{~s}^{-1}$ in the $20-100 \mathrm{keV}$ band, the PDS instrument on-board BeppoSAX offers the opportunity to study the extragalactic sky with an unprecedented sensitivity. In this work we report on the results of a search in the BeppoSAX archive for serendipitous high energy sources at high galactic latitudes $\left(|b| \geq 13^{\circ}\right)$. We have defined a set of twelve regions in which the PDS/MECS cross-calibration constant is higher than the nominal value. We attribute this mismatch to the presence of a serendipitous source in the PDS field of view. In four cases the likely high energy emitter is also present in the MECS field of view. In these cases, we have performed a broad band spectral analysis (1.5-100 keV) to understand the source spectral behaviour and compare it with previous BeppoSAX observations when available. In eight cases the identification of the source likely to provide the PDS spectrum is based on indirect evidence (extrapolation to lower energies and/or comparison with previous observations). This approach led to the discovery of six new hard X-ray emitting objects (PKS 2356-611, 2MASX J14585116-1652223, NGC 1566, NGC 7319, PKS 0101-649 and ESO 025-G002) and to the presentation the PDS spectrum of NGC 3227 for the first time. In the remaining five cases we provide extra BeppoSAX observations that can be compared with measurements already published and/or in the archive.
\end{abstract}

Key words. X-rays: general - X-rays galaxies - methods: data analysis

\section{Introduction}

The hard X-ray sky is still poorly explored at high galactic latitudes and the only truly all sky survey performed so far above $\sim 15 \mathrm{keV}$ dates back to the 1980 s (Levine et al. 1984). This pioneering work was performed with the HEAO $1 \mathrm{~A} 4$ experiment and detected $\sim 70$ sources in the $13-80 \mathrm{keV}$ band down to a flux limit of $2-3 \times 10^{-10} \mathrm{erg} \mathrm{cm}^{-2} \mathrm{~s}^{-1}$ with an angular resolution of $\sim 3^{\circ}$; of these sources sixteen were located at high galactic latitudes $\left(|b| \geq 13^{\circ}\right)$ and only seven were of extragalactic nature.

A step forward will be provided by the imager on-board INTEGRAL which is surveying a large fraction of the sky with a sensitivity better than a few $\mathrm{mCrab}^{1}$ in the $20-100 \mathrm{keV}$ energy range and an angular resolution of a few arcmin (Bassani et al. 2004; Bird et al. 2004); this exploratory work will be followed by the SWIFT mission which is expected to survey the hard X-ray sky with a sensitivity of $\sim 1 \mathrm{mCrab}$ at high galactic latitudes (Gehrels et al. 2004). In the meantime, the BeppoSAX archive can be used to probe the extragalactic X-ray sky in the same $20-100 \mathrm{keV}$ energy band. Pointed observations of BeppoSAX/PDS have unveiled many hard X-ray emitting AGN and provided the best spectroscopy of this type of object above $10 \mathrm{keV}$. However, observations were sometimes limited by the

${ }^{1}$ For a Crab-like spectrum 1 mCrab corresponds to $2 \times$ $10^{-11} \mathrm{erg} \mathrm{cm}^{-2} \mathrm{~s}^{-1}$ in the $20-100 \mathrm{keV}$ energy band. lack of imaging capability: contaminating sources were found inside the target field of view as well as in the offset fields used for background measurements. These data have often been neglected by the original observers although they provide another powerful tool with which to study the extragalactic sky above $10 \mathrm{keV}$ : new sources can be found and spectroscopically measured, while known objects can be re-observed and compared to previous measurements in a search for variability, so far poorly explored. Furthermore, the argument put forward by Fabian (2001) that two of the three nearest AGN have very high column densities $\left(>10^{24} \mathrm{~cm}^{-2}\right)$ indicates the need to constrain the statistics of highly absorbed AGN: increasing the number of such objects known is therefore an important task. In view of these facts, we have carried out a program to search systematically in the BeppoSAX/PDS archive (which at the moment is being reanalysed using the $X A S$ software package) for observations that indicate the presence of a contaminating source either in the pointed field of view (search mode 1) or in the offset fields used for background measurements (search mode 2). Results related to search mode 1 are presented in this work, while a future work will be devoted to results found in search mode 2.

The paper is organized as follows: in Sect. 2 the selected sample is described, in Sect. 3 the observations, data reduction techniques and analysis procedures are presented, while Sect. 4 
is devoted to the discussion of the results. Conclusions and future work are summarized in Sect. 5.

\section{Sample selection and contaminating source search}

Although BeppoSAX was not designed to perform an X-ray survey, a systematic search in the entire PDS data archive could allow the discovery of new sources, down to a flux limit of $\sim 10^{-11} \mathrm{erg} \mathrm{cm}^{-2} \mathrm{~s}^{-1}$. In particular, the simultaneous monitoring of three 1.3 sky regions (target field + two offset fields) allowed the PDS to survey a substantial fraction of the sky over the 15-100 keV energy range.

The PDS instrument has a hexagonal field of view of $1.3 \times 1.3 \mathrm{FWHM}$ and no imaging capability; its positional uncertainty can be approximated by an error box circle of 1.3 in radius. The MECS has instead a field of view of $30^{\prime}$ radius and so covers about $25 \%$ of the PDS area. It is therefore likely that a serendipitous source can be undetected by the MECS, but still observed by the PDS.

In this paper we concentrate on the study of those sources discovered in the pointed fields of view or detected in search mode 1 . The search in this mode was performed in the following way: first, we visually inspected in the BeppoSAX archive (wWw.asdc.asi.it) all observations performed above $13^{\circ}$ in galactic latitude and available to the public as of October 2001 (635); from this set of data we extracted a sample containing those sources which clearly show a mismatch between the MECS and PDS spectra in the standard archive analysis: this mismatch was taken as strong evidence for the presence of a contaminating source in the PDS field of view. From this preliminary list we excluded all those sources that were likely to be Compton thick on the basis of various considerations (i.e. large iron line equivalent width, $\mathrm{X}$-ray to $\left[\mathrm{O}_{\mathrm{III}}\right]$ ratio less than 1, extreme absorption): in the spectrum of these types of sources a MECS/PDS spectral mismatch is simply due to a more complex spectral shape than used for the quick look analysis. We then performed a cut in the signal to noise ratio, accepting a source only if it had at least $3 \sigma$ detection in the PDS. We then confirmed with our own analysis the MECS/PDS mismatch by fitting the MECS and PDS data with a simple model, generally a power law either unabsorbed or absorbed: only when the cross-calibration constant between these two instruments was significantly outside the nominal range of $0.75-0.95$ (Fiore et al. 1999a), i.e. greater than 2, was a source maintained. Overall, this analysis provided the sample of twelve regions discussed in the present paper. Although this sample is not complete (not all observations performed by BeppoSAX were screened), this search gives an idea of the extragalactic sources that populate the hard X-ray sky; in this sense the sources serendipitously found in this work can be "loosely" taken as representative of the AGN population in the 20-100 keV band.

An important step in the search described above is the reduction of the PDS spectra extracted using the XAS v2.1 package (Chiappetti \& Dal Fiume 1997): this provides slightly different results than $S A X D A S$, i.e. the package usually used to perform the archive analysis. A preliminary comparison between PDS spectra extracted by means of these two software packages is under way ${ }^{2}$ and will be presented in a future work. The analysis performed on sources of different intensity show that the spectral parameters do not change when computed with the different packages, but the associated errors are smaller when using XAS. A significant improvement in the signal to noise ratio is obtained by means of $X A S$. Furthermore, the $X A S$ package allows a more reliable check of the background fields by taking advantage of the rocking technique (Frontera et al. 1997b); this is important when the source is faint in the PDS as is often our case. When one collimator is pointing $\mathrm{ON}$ source, the other collimator is pointing in one of the two OFF positions. The standard stay time in each position of either collimator was $96 \mathrm{~s}$. At each cycle the two collimators were swapped: the one pointing to the source was moved to monitor the background and vice versa. In this way we can obtain, in addition to the target observation, two independently accumulated spectra of the two +OFF and -OFF fields offset by $210^{\prime}$ with respect to the main pointing; these offsets are used as background in the computation of the spectrum of the target source. The comparison between the spectra of these two offset fields and, in particular, the difference between the +OFF and -OFF spectra in count rate is a good diagnostic tool to investigate the presence of contamination.

If no contamination is present in either of the offset fields, we expect the difference of their count rates to be compatible with zero; on the contrary, a positive excess of counts in the difference indicates the presence of contamination in the $+\mathrm{OFF}$ field; a negative count rate provides evidence for contamination in the-OFF field.

Applying this method to our sample, we found the presence of a contaminating source in the +OFF field of the first observation of AD Leonis (excess: $0.189 \pm 0.070$ counts s $^{-1}, 2.7 \sigma$ ) and in part of the first observation of VW Cephei (excess: $0.139 \pm$ 0.043 counts $\left.^{-1}, 3.2 \sigma\right)$, while in part of the observations of MKN 1073 and NGC 7552 the -OFF fields show an excess of $0.159 \pm 0.060$ counts s $^{-1}(2.7 \sigma)$ and $0.203 \pm 0.0 .056$ counts s $^{-1}$ $(3.6 \sigma)$, respectively. To give an idea of the effect of contamination in an offset field on the PDS data, we have considered two extreme cases: a $\sim 3 \sigma$ excess reduces the source count rate by $\sim 5 \%$, while a $10 \sigma$ contamination provides a reduction of around $15 \%$.

In order to extract the uncontaminated source spectra, we excluded the contaminated fields and considered only the uncontaminated ones in the computation of the background for these particular sources.

After assembling the sample, we search for the likely contaminating sources, adopting the following strategy. First, we considered all the sources present in the MECS image in addition to the target source and checked their consistency with the PDS data, i.e. if the cross-calibration constant fell within the nominal interval. If no sources in the MECS field of view matched the PDS data, then, we searched for likely high energy emitters located inside the PDS field of view but not observed

\footnotetext{
2 On behalf of the PDS group, see

ftp://ftp.tesre.bo.cnr.it in the directory /pub/sax/doc/ software_docs/xas_vs_saxdas.ps
} 
Table 1. The list of serendipitous PDS detections found in the BeppoSAX archive.

\begin{tabular}{|c|c|c|c|c|c|}
\hline $\begin{array}{l}\text { Pointed } \\
\text { Source }\end{array}$ & Obs. date & Type & $\mathrm{Calib}^{(a)}$ & $\begin{array}{l}\text { Contaminating } \\
\text { Source }\end{array}$ & Type \\
\hline IRAS 01025-6423 & $24 / 03 / 2001$ & Seyfert 2 & $18-1324$ & PKS 0101-649 & Quasar \\
\hline MKN 1073 & $15 / 02 / 1999$ & Seyfert 2 & $485-2035$ & Perseus/NGC 1275 (?) & Cluster/Seyfert 2 \\
\hline NGC 1553 & $\begin{array}{l}16 / 01 / 1997 \\
16 / 11 / 1997\end{array}$ & Normal Galaxy & $14-46$ & NGC 1566 & Seyfert 1 \\
\hline AD Leonis & $\begin{array}{l}23 / 04 / 1997 \\
01 / 05 / 1999 \\
08 / 05 / 1999 \\
12 / 05 / 1999\end{array}$ & Flare Star & $152-230$ & NGC 3227 & Seyfert 1 \\
\hline ON 325 & $23 / 12 / 1998$ & Blazar & $11-57$ & MKN $766^{(b)}$ & Seyfert 1 \\
\hline NGC 5793 & $25 / 07 / 2001$ & Seyfert 2 & $32-1384$ & 2MASX J14585116-165 & Galaxy \\
\hline 1E $1839.6+8002$ & $\begin{array}{l}16 / 10 / 2000 \\
03 / 02 / 2001\end{array}$ & Flare Star & $83-181$ & $3 \mathrm{C} 390.3^{(b)}$ & Seyfert 1 \\
\hline H1846-786 & 08/03/2001 & Seyfert 1 & $2-4$ & ESO 025-G002 & Seyfert 1 \\
\hline VW Cephei & $\begin{array}{l}07 / 05 / 1998 \\
07 / 10 / 1998\end{array}$ & Eclipsing Binary & $164-417$ & $4 \mathrm{C}+74.26$ & Quasar \\
\hline NGC 7331 & $10 / 06 / 2000$ & LINER & $28-121$ & NGC 7319 & Seyfert 2 \\
\hline NGC 7552 & 23/12/1999 & Galaxy & $150-309$ & NGC 7582 & Seyfert 2 \\
\hline SCG 2353-6101 & 28/11/1996 & Cluster & $8-28$ & PKS $2356-611^{(b)}$ & Seyfert 2 \\
\hline
\end{tabular}

by the MECS given the significantly different fields of view. In particular, when adopting this second approach, we focused our search on bright sources in the $2-10 \mathrm{keV}$ band as these are the most likely to contaminate the PDS observation and are expected to appear in the HEASARC X-ray archives. In Table 1 we list the twelve cases we found, reporting in each case the BeppoSAX observation target with its relative observation date and object type, the PDS/MECS cross-calibration constant obtained by fitting the data with a simple power law and finally the name and type of the contaminating source found. It is evident from the Table that the cross-calibration constant is always outside the nominal range, confirming the presence of one or more contaminating objects in the PDS field of view. Extrapolation of a more complex model from the MECS to the PDS energy range still provides in all cases a high cross calibration constant. We found only four fields where the contaminating source is so close $\left(\sim 25^{\prime}\right)$ to theBeppoSAX target that it is also detected by the MECS instrument.

\section{Observations and data analysis}

In this work we made use of data from three of the Narrow Field Instruments (NFIs) on-board the Italian-Dutch satellite BeppoSAX (Boella et al. 1997a): the Low Energy Concentrator Spectrometer (LECS, 0.1-10 keV, Parmar et al. 1997), the Medium Energy Concentrator Spectrometer (MECS, 1.3-10 keV, Boella et al. 1997b) and the Phoswich Detection System (PDS, 15-300 keV, Frontera et al. 1997b).
For all sources, the LECS and MECS spectra were downloaded from the BeppoSAX archive; the analysis of the on-axis source was standard. For all the off-axis sources detected in the MECS field of view, contaminating or not, the MECS spectra were extracted from a region centered on the source and having a radius chosen according to the criteria suggested by Fiore et al. (1999a). For these sources, because of the lack of the appropriate ancillary response files, the LECS spectral analysis could not be performed. The background subtraction for the on-axis sources was performed using blank sky spectra extracted from the same region of the source, while for the offaxis sources we used a local background spectrum (extracted from a region with a radius equal to the source extraction radius) to account for possible contaminating effects inside the MECS field of view.

For the PDS data reduction, source visibility windows were selected following the criteria of no Earth occultation and high voltage stability during the exposure. In addition, the observations closest to the South Atlantic Anomaly were discarded from the analysis.

The LECS and MECS spectra were rebinned in order to sample the energy resolution of the detectors with an accuracy proportional to the count rate. The PDS data were instead rebinned to have logarithmically equal energy intervals. The data rebinning also required that there were at least 20 counts in each bin so that the $\chi^{2}$ statistic could reliably be used.

The energy bands used for spectral fitting were limited to those where the response functions are well known, i.e. 0.1-4.5 keV, 1.5-10.5 keV and 15-100 keV, for the LECS MECS and PDS, respectively. 
Table 2. Contribution to the PDS flux of the sources detected in the MECS field of view.

\begin{tabular}{llcccc}
\hline \hline Field & Source & $\begin{array}{c}\text { Extrapolated flux } \\
{[20-100] \mathrm{keV}}\end{array}$ & $\begin{array}{c}\text { Contribution }^{(\%)} \\
{\left[\begin{array}{c}\text { Offset }^{(b)} \\
(\operatorname{arcmin})\end{array}\right.}\end{array}$ & $\begin{array}{c}R^{(c)} \\
(\%)\end{array}$ \\
\hline ON 325 & ON 325 & 1.2 & 10 & - & - \\
& 1AXG J121854+2957 & 0.56 & 3 & 16.2 & 78 \\
& MKN 766 & 17.5 & 87 & 19.7 & 73 \\
NGC 5793 & NGC 5793 & 0.072 & 1 & - & - \\
& NGC 5796 & 0.17 & 2 & 4.2 & 92 \\
& 2MASX J1458-165 & 7.72 & 95 & 13.4 & 81 \\
1E 1839.6+8002 & 1E 1839.6+8002 & 0.017 & $<1$ & - & - \\
& 3C 390.3 & 47.8 & 98 & 25.3 & 65 \\
SCG 2353-6101 & SCG 2353-6101 & 0.03 & $<1$ & - & - \\
& PKS 2356-611 & 25.2 & 99 & 16.1 & 78 \\
\hline
\end{tabular}

\footnotetext{
(a) In units of $10^{-12} \mathrm{erg} \mathrm{cm}^{-2} \mathrm{~s}^{-1}$.

(b) Distance between the pointing target and the off-axis sources.

(c) Source flux correction factor due to the PDS response to source an off-axis source.
}

The spectral analysis was performed using the XSPEC V11.2.0 software package (Arnaud 1996) and the instrument response matrices released by the BeppoSAX ASI Science Data Center. For the off-axis sources, we used the appropriate MECS ancillary response files to correct for the effects of vignetting due to the mirrors. In addition, we introduced a flux correction factor in the PDS band for each contaminating source in order to estimate the real flux at the source. This correction is simply a function of the distance of the source from the main target and is related to the PDS response: the reduction in sensitivity is of a factor of $\sim 2$ at $38^{\prime}$ from the main pointing coordinates while at $78^{\prime}$ the response is zero (Frontera et al. 1997a).

Normalization constants have also been introduced to allow for known differences in the absolute cross-calibration between the detectors. The LECS/MECS cross-calibration constant was allowed to lie within the nominal range 0.7-1.0 (Fiore et al. 1999a), while the PDS/MECS cross-calibration was left free to vary in order to search for the presence of contaminating sources and then to estimate their broad band spectrum. The absorption of X-rays due to our galaxy in the direction of each object (Dickey \& Lockman 1990) is added in all models of the spectral analysis. All quoted errors correspond to the $90 \%$ confidence interval for one interesting parameter $\left(\Delta \chi^{2}=2.71\right)$.

The main aim of this work is to estimate the PDS spectrum of each serendipitous source found. The method we used to achieve this was to fit simultaneously the LECS (not always available) and MECS data of the target source to determine the best-fit model to these data; afterwards, we performed an extrapolation of this best-fit model to the PDS energy band in order to estimate the contribution of the target source to the total PDS flux. Hence, by subtracting from the total PDS spectrum the contribution due to the on-axis source, we were able to estimate the high energy spectrum of the serendipitous/contaminating object.

Four sources in our sample (NGC 1553, 1E 1839.6+8002, VW Cephei and AD Leonis), have been observed by
BeppoSAX at different epochs (see Table 1). As a first step, our approach was to analyse each single pointing, for each source, individually. Although in some cases we found evidence for flux variability in the target and/or the serendipitous sources, the spectral parameters were always consistent within the respective uncertainties; therefore, we decided to sum, in all cases, all available pointings to improve the statistical quality of the data, reporting only the averaged spectral parameters.

\section{Results}

In the following, we briefly describe the main results obtained for each field. We discuss separately the four fields in which the contaminating object is detected in the MECS instrument because for these objects we can perform a broad band spectral analysis (1.5-100 keV), while in the remaining eight cases we can only present and discuss the PDS spectrum.

\subsection{Sources detected in the MECS field of view}

In this section, we describe the four fields where the object likely to be responsible for the PDS spectrum is also observed by the MECS instrument. In Table 2 (and also in Table 5 afterwards) we report for each of these fields all sources detected by the MECS, their fluxes extrapolated from the MECS to the PDS energy range (15-100 keV) assuming their best fit model, their relative contribution (in \%) to the PDS flux, their distance from the target source and the relative flux correction factor, $R$. The observed flux is divided by $R$ to reconstruct the flux at the source. It is evident from the values reported in Col. 4 (Contribution) which source is in each case responsible for most of the PDS flux. In Table 3 we list for each of these likely associations the MECS and PDS exposures and count rates, the 2-10 keV flux, the observed high energy flux $(20-100 \mathrm{keV})$ and the effective flux $(20-100 \mathrm{keV})$ at the source. For these four sources we jointly fitted the MECS and PDS data to provide a broad band (20-100 keV) spectrum. In Table 4 we summarize the best-fit parameters and also report the range of the 
Table 3. MECS and PDS observation log.

\begin{tabular}{|c|c|c|c|c|c|c|c|}
\hline \multirow[t]{2}{*}{$\overline{~ S o u r c e}$} & \multicolumn{2}{|r|}{ MECS } & \multicolumn{2}{|r|}{ PDS } & \multirow{2}{*}{$\begin{array}{c}F^{(a)} \\
{[2-10] \mathrm{keV}}\end{array}$} & $F_{\text {Obs }}^{(a)}$ & \multirow{2}{*}{$\begin{array}{c}F_{\text {Source }}^{(a)} \\
{[20-100] \mathrm{keV}}\end{array}$} \\
\hline & $\begin{array}{c}\text { EXPO } \\
(\mathrm{ks})\end{array}$ & $\mathrm{cts} / \mathrm{s}$ & $\begin{array}{c}\text { EXPO } \\
(\mathrm{ks})\end{array}$ & $\mathrm{cts} / \mathrm{s}$ & & [20-100] keV & \\
\hline MKN 766 & 31.4 & $0.0153 \pm 0.0011$ & 15.7 & $0.152 \pm 0.050$ & 16.0 & 17.0 & 23.3 \\
\hline 2MASX J1458-165 & 78.7 & $0.0042 \pm 0.0003$ & 38.5 & $0.086 \pm 0.029$ & 0.93 & 8.1 & 11.1 \\
\hline $3 C 390.3^{(b)}$ & 157.3 & $0.078 \pm 0.001$ & 80.0 & $0.447 \pm 0.002$ & 23.0 & 22.0 & 43.0 \\
\hline PKS 2356-611 & 23.4 & $0.029 \pm 0.001$ & 25.4 & $0.205 \pm 0.041$ & 13.0 & 25.5 & 32.7 \\
\hline
\end{tabular}

Note: Count rates are referred to the energy bands used in the spectral analysis (see text).

(a) In units of $10^{-12} \mathrm{erg} \mathrm{cm}^{-2} \mathrm{~s}^{-1}$.

(b) For this source the parameters of the average spectrum are reported.

Table 4. MECS and PDS spectral analysis of the serendipitous sources detected within the MECS field of view.

\begin{tabular}{lcccccc}
\hline \hline Source & $\Gamma$ & $N_{\mathrm{H}}^{(a)}$ & $\begin{array}{c}E_{\text {Line }} \\
(\mathrm{keV})\end{array}$ & $\begin{array}{c}E W \\
(\mathrm{eV})\end{array}$ & $\mathrm{Calib}^{(b)}$ & $\chi^{2} / v$ \\
\hline MKN 766 & $1.93 \pm 0.10$ & - & - & - & $0.5-1.3$ & $36.4 / 36$ \\
2MASX J1458-165 & $1.20_{-0.23}^{+0.26}$ & - & - & - & $0.8-3.2$ & $16.1 / 24$ \\
3C 390.3 ${ }^{(c)}$ & $1.72_{-0.05}^{+0.04}$ & $<0.08$ & $6.37_{-0.25}^{+0.29}$ & $115_{-84}^{+86}$ & $0.89-1.22$ & $16.5 / 22$ \\
PKS 2356-611 & $1.70_{-0.17}^{+0.18}$ & $10.2_{-2.31}^{+2.91}$ & $6.46_{-0.17}^{+0.21}$ & $523_{-246}^{+238}$ & $0.75-0.95$ & $48.5 / 41$ \\
\hline
\end{tabular}
(a) In units of $10^{22} \mathrm{~cm}^{-2}$
(b) PDS/MECS cross-calibration constant.
${ }^{(c)}$ For this source the parameters of the average spectrum are reported.

Table 5. Contribution to the PDS flux of all the MECS sources in the eight fields in which the contaminating source was not detected in the MECS field of view.

\begin{tabular}{llcccc}
\hline \hline Field & Source & $\begin{array}{c}\text { Extrapolated flux } \\
{[20-100] \mathrm{keV}}\end{array}$ & $\begin{array}{c}\text { Contribution } \\
(\%)\end{array}$ & $\begin{array}{c}\text { Offset }^{(b)} \\
(\operatorname{arcmin})\end{array}$ & $\begin{array}{c}R^{(c)} \\
(\%)\end{array}$ \\
\hline IRAS 010125-6423 & IRAS 010125-6423 & 0.002 & $<1$ & - & - \\
MKN 1073 & MKN 1073 & - & - & - & - \\
NGC 1553 & NGC 1553 & 1.5 & 7 & - & - \\
Ad Leonis & AD Leonis & 0.0002 & $<1$ & - & - \\
H1846-786 & H1846-786 & 10 & 50 & - & - \\
VW Cephei & VW Cephei & 0.0004 & $<1$ & - & - \\
NGC 7331 & NGC 7331 & 0.59 & 4 & - & - \\
NGC 7552 & NGC 7552 & 0.12 & $<1$ & - & - \\
& FRL 1041 & 1.78 & 4 & 18.9 & 76 \\
& Sersic 159-03 & 0.003 & $<1$ & 25.8 & 65 \\
\hline
\end{tabular}

\footnotetext{
(a) In units of $10^{-12} \mathrm{erg} \mathrm{cm}^{-2} \mathrm{~s}^{-1}$.

(b) Distance between the pointing target and the off-axis sources.

${ }^{(c)}$ Source flux correction factor due to the PDS response to source not on-axis.
}

cross-calibration constant PDS/MECS (Col. 6). As is evident from the values of Col. 6 , the normalization constants obtained are always compatible with the suggested value of $0.75-0.95$. This is a further indication that these sources are indeed responsible for the high energy emission detected by the PDS instrument.

\subsubsection{The ON 325 field}

Within the MECS field of view (see Fig. 1) of this pointing we find two other sources besides the target. The first, located at $\alpha(2000)=12^{\mathrm{h}} 18^{\mathrm{m}} 54^{\mathrm{s}} .4$ and $\delta(2000)=+29^{\circ} 58^{\prime} 10^{\prime} .5$, is identified with the Seyfert 1.9 galaxy 1AXG J121854+2957, which belongs to the ASCA Medium Sensitivity Survey (AMSS) (Ueda et al. 2001) and to the BeppoSAX High Energy Large Area Survey (HELLAS) (Fiore et al. 1999b). The second is located at $\alpha(2000)=12^{\mathrm{h}} 18^{\mathrm{m}} 25^{\mathrm{s}} .2$ and $\delta(2000)=+29^{\circ} 48^{\prime} 48^{\prime \prime} .9$, $\sim 20^{\prime}$ from ON 325 and corresponds to the Seyfert 1 galaxy MKN 766.

Following Perri et al. (2003), the LECS and MECS data of the blazar ON 325 are best fitted with a broken power law, for which we obtain a $\chi^{2} / v=32.4 / 25$ and a $2-10 \mathrm{keV}$ flux of $\sim 8 \times 10^{-13} \mathrm{erg} \mathrm{cm}^{-2} \mathrm{~s}^{-1}$. The two power laws intersect at $E_{B}=$ $4.12_{-0.83}^{+1.10} \mathrm{keV}$ and have photon indices $\Gamma_{1}=2.41_{-0.27}^{+0.18}$ and $\Gamma_{2}=$ $0.44_{-0.85}^{+0.79}$ in agreement with Perri et al. (2003). Adopting this 


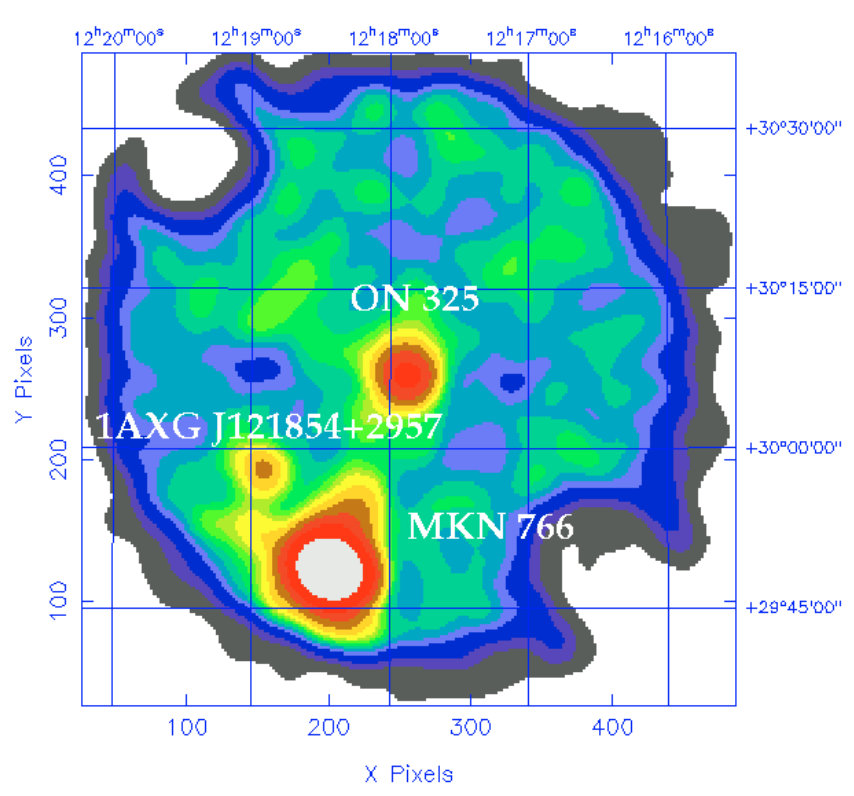

Fig. 1. MECS image of the sky region surrounding ON 325.

model, we estimate a contribution of ON 325 to the PDS data of the order of $10 \%$ (see Table 2).

The MECS spectra of 1AXG J121854+2957 is instead well fitted $\left(\chi^{2} / v=10.8 / 14\right)$ with an absorbed power law having a photon index $\Gamma \sim 2.3$, a column density $N_{\mathrm{H}}<12 \times 10^{22} \mathrm{~cm}^{-2}$ and a $2-10 \mathrm{keV}$ flux of $8.5 \times 10^{-13} \mathrm{erg} \mathrm{cm}^{-2} \mathrm{~s}^{-1}$. These values are in agreement with those found by Loaring et al. (2003) during a $X M M-N e w t o n$ observation of the source. Extrapolation of this spectrum to the PDS band indicates a small contribution to the high energy flux, while a simultaneous fit to the MECS/PDS data provides a PDS/MECS cross-calibration constant in the range 2-30, i.e. well outside the nominal interval. A good fit $\left(\chi^{2} / v=30 / 34\right)$ of the MECS data of MKN 766 is also described by a simple power law with a photon index $\Gamma=$ $1.92 \pm 0.13$ and a $2-10 \mathrm{keV}$ flux of $1.7 \times 10^{-11} \mathrm{erg} \mathrm{cm}^{-2} \mathrm{~s}^{-1}$. The high contribution to the PDS flux (see Table 2) found with this model indicates that MKN 766 is the best candidate to account for the PDS flux. In fact, the fit of the MECS spectrum of MKN 766 with the PDS data gives a PDS/MECS cross-calibration constant well inside the nominal interval. The $2-10 \mathrm{keV}$ flux (see Table 3), found during this pointing, is slightly lower than the value of $2.05 \times 10^{-11} \mathrm{erg} \mathrm{cm}^{-2} \mathrm{~s}^{-1}$ found by Matt et al. (2000a) during a previous (May 1997) BeppoSAX dedicated observation. We find no evidence for an iron line at around $6.4 \mathrm{keV}$ (see Fig. 2), consistent with the fact that this feature seems to be present only when MKN 766 is in a high state (Leighly et al. 1996).

To confirm if MKN 766 is really the contaminating source in the ON 325 field, we also reanalysed the PDS data of the two pointings of MKN 766 performed by BeppoSAX in May 1997 and 2001. In the first observation the comparison between the $+\mathrm{OFF}$ and $-\mathrm{OFF}$ field count rates shows an excess of $3 \sigma$ in the -OFF field, while in the second there is no evidence for contamination.

Searching in the HEASARC archive for possible objects which could contaminate the -OFF field, we found two

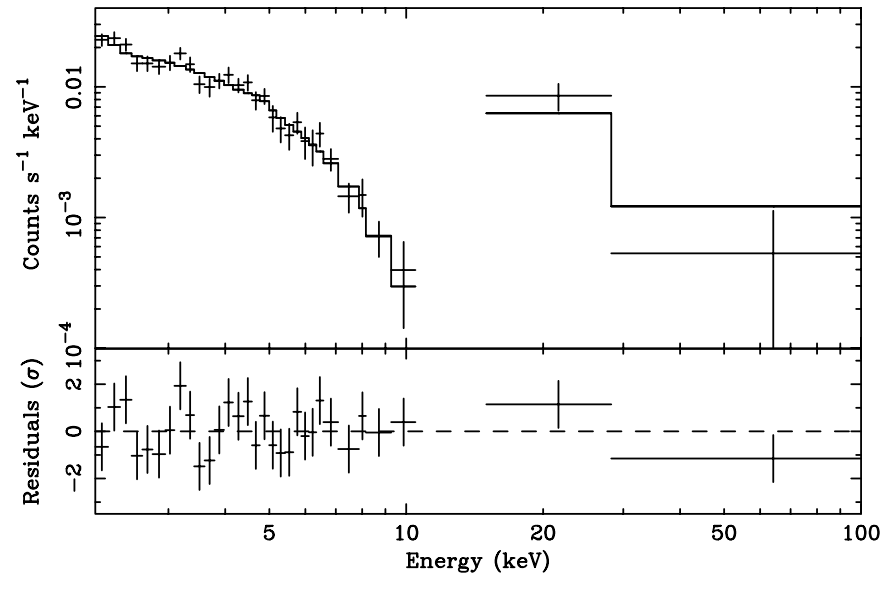

Fig. 2. Broad band spectrum of MKN 766 fitted with a simple power law (upper panel) and residuals to this model in units of $\sigma$ (lower panel). The data are graphically rebinned.

sources belonging to the ROSAT Bright Source Catalog: a quasar (1RSX J121320.3+270841) and an unidentified object (1RSX J121258.3+272653), at a distance of $\sim 40^{\prime}$ and $\sim 59^{\prime}$ from the field centre. Fitting the -OFF data with a simple power law, we obtain $\Gamma \leq 2$ and a $20-100 \mathrm{keV}$ flux of $3.6 \times 10^{-11} \mathrm{erg} \mathrm{cm}^{-2} \mathrm{~s}^{-1}$. Since both sources have similar count rates in ROSAT, we can only state that either or both could be responsible for this excess.

This provides a good example of the capability of our analysis: contamination in the offset fields can be found and identified, while at the same time a more correct evaluation of the target spectrum is provided.

The PDS spectrum of MKN 766 in the May 1997 observation can be corrected and reanalysed together with that of May 2001. In both cases the photon index $\Gamma$ is $\sim 2$, in agreement with that found in December 1998 (i.e. during the ON 325 observation), while the $20-100 \mathrm{keV}$ flux is roughly $\sim 3 \times 10^{-11} \mathrm{erg} \mathrm{cm}^{-2} \mathrm{~s}^{-1}$, higher than during the ON 325 measurement; this finding indicates that the flux variability always seen in this source at soft X-ray energies (Matt et al. 2000a and references therein) extends to above $\sim 10 \mathrm{keV}$.

\subsubsection{The NGC 5793 field}

NGC 5793 has been detected at $\sim 5 \sigma$ level in the MECS instrument, while it is insignificant $(<1 \sigma)$ in the LECS energy range. Fitting the MECS data with a simple power law of photon index $\Gamma=1.9$ we obtain a very low flux of $\sim 7 \times 10^{-14} \mathrm{erg} \mathrm{cm}^{-2} \mathrm{~s}^{-1}$. This model, extrapolated to the high energy band, gives a negligible contribution to the PDS data (see Table 2).

Inspection of Fig. 3 indicates the presence of two extra sources in the MECS field of view. The first located at $\alpha(2000)=14^{\mathrm{h}} 58^{\mathrm{m}} 50^{\mathrm{s}} .7$ and $\delta(2000)=-16^{\circ} 51^{\prime} 50.7$, corresponds to a bright irregular spiral galaxy belonging to the 2 Micron All Sky Survey eXtended Source Catalog (i.e. 2MASX J14585116-1652223). The second is located at $\alpha(2000)=14^{\mathrm{h}} 59^{\mathrm{m}} 23^{\mathrm{s}} .8$ and $\delta(2000)=-16^{\circ} 37^{\prime} 13^{\prime \prime} .4$ and is identified with the normal galaxy NGC 5796. 


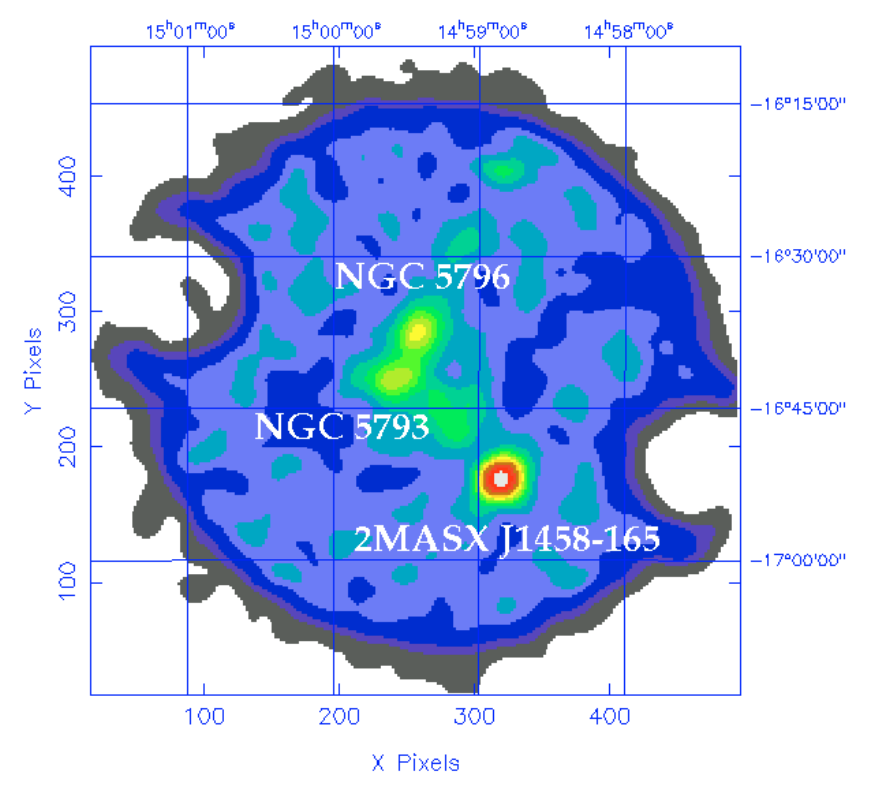

Fig. 3. MECS image of the sky region surrounding NGC 5793.

We extracted the MECS spectra of both sources and extrapolated them to high energies. We find that the MECS spectrum of NGC 5796 is well fitted $\left(\chi^{2} / v=15 / 20\right)$ with a simple power law having a photon index $\Gamma=1.95_{-0.56}^{+0.67}$ and a $2-10 \mathrm{keV}$ flux of $1.5 \times 10^{-13} \mathrm{erg} \mathrm{cm}^{-2} \mathrm{~s}^{-1}$. The contribution of NGC 5796 to the PDS flux is only a few percent and the PDS/MECS crosscalibration constant is extremely high (20-103).

The MECS best-fit model in the case of 2MASX J14585116-1652223 is a simple power law with photon index $\Gamma=1.15_{-0.18}^{+0.33}$ and a $2-10 \mathrm{keV}$ flux of $9.3 \times 10^{-13} \mathrm{erg} \mathrm{cm}^{-2} \mathrm{~s}^{-1}\left(\chi^{2} / v=15 / 20\right)$. The extrapolation of this model to high energies (see Table 2) suggests that 2MASX $\mathrm{J} 14585116-1652223$ is likely to be the contaminating object. The simultaneous fit of the MECS/PDS data (see Table 3 and Fig. 4) shows a flat spectrum $\left(\Gamma=1.20_{-0.23}^{+0.26}\right)$, a more suitable value (0.7-3.5) for the PDS/MECS cross-calibration constant and no evidence for extra absorption. Fixing $\Gamma=1.9$ and adding absorption to the power law provides a similar fit and an upper limit to the column density of $\sim 4 \times 10^{22} \mathrm{~cm}^{-2}$. As displayed in Table 3, the $20-100 \mathrm{keV}$ flux turns out to be of $1.11 \times 10^{-11} \mathrm{erg} \mathrm{cm}^{-2} \mathrm{~s}^{-1}$, one order of magnitude higher than the 2-10 keV flux, confirming that 2MASX J145851161652223 emits mostly in the high energy band. Very little is known about this object except for its near infrared properties: the source is fairly bright with a total photometry of 14.4, 13.6 and 13 mag in the $J, H$ and $K$ bands respectively and an extent of $11^{\prime \prime}$; the optical counterpart has magnitudes $B=16$ and $R=15$. No previous X-ray data are reported, including the lack of detection by ROSAT, which would be consistent with the presence of strong absorption. The $R-K$ colour is $\sim 2$, i.e. quite red which again suggests an object with considerable extinction. No detection in the radio band was found in the literature or in the HEASARC archive.

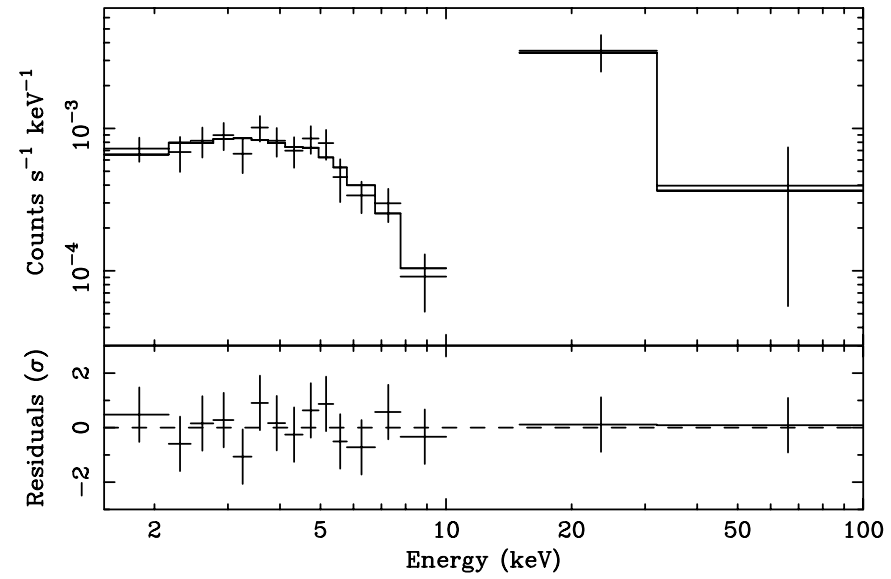

Fig. 4. Broad band spectrum of 2MASX J1458-165 fitted with a simple power law (upper panel) and residuals to this model in units of $\sigma$ (lower panel). The data are graphically rebinned.

\subsubsection{The $1 \mathrm{E} 1839.6+8002$ field}

The M4 Ve star 1E 1839.6+8002 was observed by BeppoSAX twice (October 2000 and February 2001). This source was detected for the first time during the Einstein observation of the Broad Line Radio Galaxy 3C 390.3 in the 1980s. A detailed study of the spectral behaviour of this source is beyond the aim of this paper. Nevertheless, following Pan et al. (1997), the LECS and MECS spectra of both observations are well described by a thermal model (RAYMOND-SMITH model in XSPEC, Raymond \& Smith 1977), with a temperature $k T \sim 1.8 \mathrm{keV}$ and a $2-10 \mathrm{keV}$ flux of $9.5 \times 10^{-14} \mathrm{erg} \mathrm{cm}^{-2} \mathrm{~s}^{-1}$ (first observation) and $7.2 \times 10^{-14} \mathrm{erg} \mathrm{cm}^{-2} \mathrm{~s}^{-1}$ (second observation).

The source appears to be faint and variable $(\sim 25 \%)$ in the $2-10 \mathrm{keV}$ range, with a negligible contribution to the high energy emission in both observations (in Table 2 only the averaged contribution of the source is reported).

Within the MECS field of view (see Fig. 5) of both measurements, a bright object located at $\alpha(2000)=18^{\mathrm{h}} 41^{\mathrm{m}} 51^{\mathrm{s}} .5$ and $\delta(2000)=+79^{\circ} 47^{\prime} 01^{\prime \prime} .3$ is clearly visible: it corresponds to the well-known radio galaxy 3C 390.3. The Einstein IPC data of 3C 390.3 revealed the presence of strong intrinsic absorption (Kruper et al. 1990), while at higher energies Ginga data showed the presence of an iron line at $6.4 \mathrm{keV}$ (Inda et al. 1994) and a reflection component (Nandra \& Pounds 1994). ASCA data confirmed the presence of the iron $K$ emission line but could not constrain the reflection hump, most probably because of the limited energy range (Eracleous et al. 1996; Leighly et al. 1997). 3C 390.3 has also been detected by $O S S E$ in the soft- $\gamma$ energy range, above $50 \mathrm{keV}$ (Dermer \& Gherels 1995). All these results have been confirmed by Grandi et al. (1999), on the basis of a dedicated BeppoSAX observation performed in January 1997. The above authors also found a column density variability when comparing BeppoSAX results with previous measurements.

No evidence for variability is present in the MECS data of 3C 390.3 nor in the PDS analysed in this work. Hence, in order 


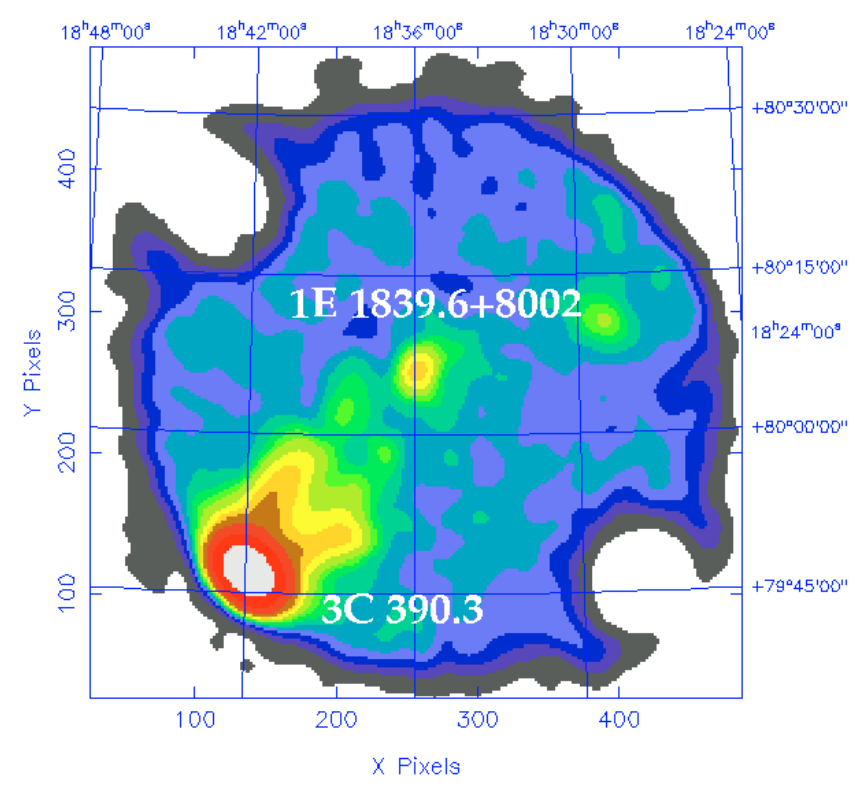

Fig. 5. MECS image of the sky region surrounding 1E 1839.6+8002.

to improve the statistics, we summed the two data sets to perform our spectral analysis. First, we analysed only the MECS data of $3 \mathrm{C} 390.3$, finding as best-fit model $\left(\chi^{2} / v=13.5 / 17\right)$ a power law with a slope of 1.7 plus a narrow Gaussian line having a centroid energy of $6.4 \mathrm{keV}$ and an equivalent width of $E W=115 \mathrm{eV}$, compatible with $\mathrm{K}_{\alpha}$ line emission from neutral iron located at the source redshift. The contribution of the 3C 390.3 flux to the total PDS flux is significant (see Table 2) and clearly indicates that this source is indeed responsible for the contamination.

The joint fit of the MECS data of this source with the PDS is also well reproduced by a power law plus a narrow Gaussian line $\left(\chi^{2} / v=16.5 / 22\right)$ having parameters similar to those found fitting only the MECS data. As for the column density, we can only provide an upper limit of $\sim 8 \times 10^{20} \mathrm{~cm}^{-2}$, compatible with the values found and reported by Grandi et al. (1999). The broad band spectrum of the source is shown in Fig. 6. The flux turns out to be $2.2 \times 10^{-11} \mathrm{erg} \mathrm{cm}^{-2} \mathrm{~s}^{-1}$ and $4.3 \times 10^{-11} \mathrm{erg} \mathrm{cm}^{-2} \mathrm{~s}^{-1}$ in the 2-10 and 20-100 keV band respectively, again in agreement with that found by Grandi et al. (1999). The PDS/MECS cross-calibration constant turns out to be consistent (see Table 4) with the suggested values, confirming that 3C 390.3 is the serendipitous source contaminating the PDS emission during the observations of the 1E 1839.6+8002 field of view (see Table 2). There is no evidence for variability between the present (2000-2001) BeppoSAX observations and that reported by Grandi et al. (1999) in the 1997 observation.

\subsubsection{The SCG 2353-6101 field}

In the case of the cluster of galaxies SCG 2353-6101 (Abell 4067) there is another source in the MECS field of view, PKS 2356-611, located at $\alpha(2000)=23^{\mathrm{h}} 59^{\mathrm{m}} 04^{\mathrm{s}} .1$ and $\delta(2000)=-60^{\circ} 55^{\prime} 01^{\prime \prime} .5$ (see Fig. 7).

PKS 2356-611 $(z=0.096, \mathrm{~B}=12.3, \mathrm{R}=12.8$, as reported in the USNO-B1 Catalog (Monet et al. 2003)) is one of

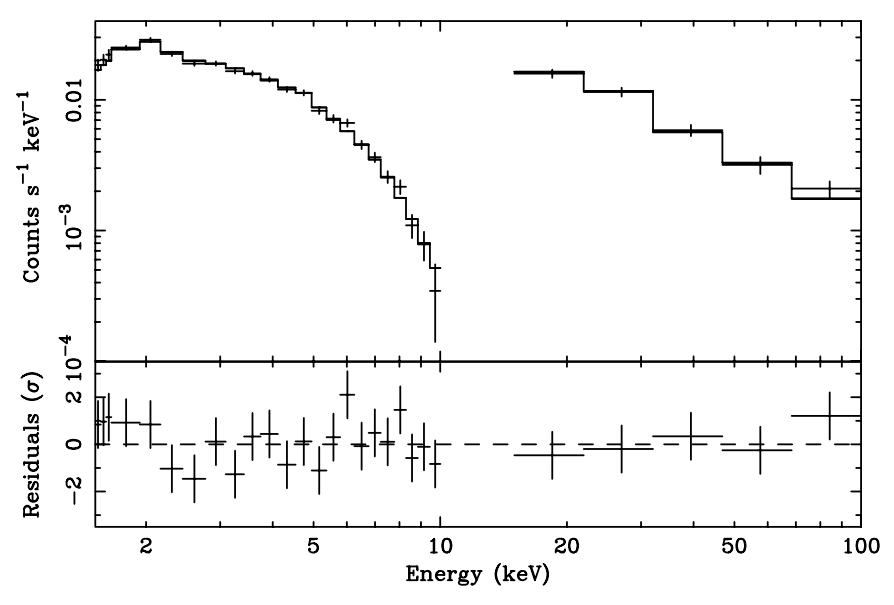

Fig. 6. Broad band spectrum of 3C 390.3 fitted with an absorbed power law plus a narrow Gaussian line (upper panel) and residuals to this model in units of $\sigma$ (lower panel).

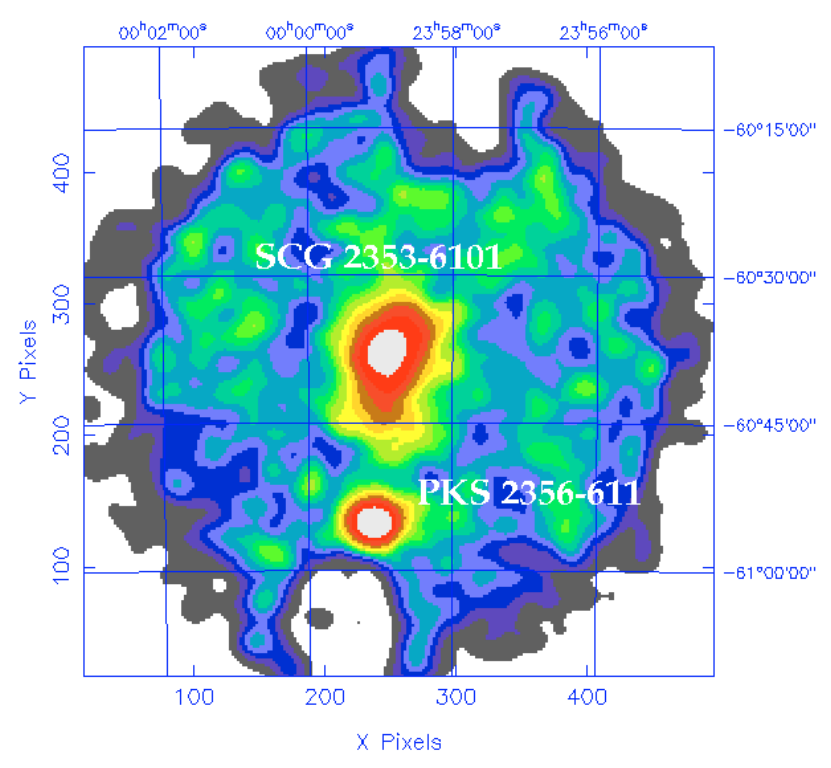

Fig. 7. MECS image of the sky region surrounding SCG 2353-6101.

the strongest southern FR II sources, with a total radio power $P_{1.4 \mathrm{GHz}} \sim 10^{25.8} \mathrm{~W} \mathrm{~Hz}^{-1}$ (Koekemoer et al. 1998), while optically it shows strong high-excitation narrow line emission with $\left[\mathrm{O}_{\text {III }}\right] \lambda 5007 / \mathrm{H}_{\beta}>10$. Lipovetsky et al. (1988) have cataloged this source as a Seyfert galaxy of type 2. It is also listed in the 2 Micron All Sky Survey eXtended Source Catalog as 2MASX J23590436-6054594 (with magnitudes $J=13.7$, $H=12.7, K=12.9$ ) and was not detected by ROSAT.

The LECS and MECS spectra of SCG 2353-6101 are well fitted $\left(\chi^{2} / v=32 / 37\right)$ with a thermal bremsstrahlung model, giving a temperature of $4.74_{-2.26}^{+1.70} \mathrm{keV}$. The extrapolation of this model to the PDS energy band provides negligible flux (see Table 2). This, combined with the high value (8-28) of the PDS/MECS cross-calibration, indicates the presence of a high energy emitting source and PKS 2356-611 is a good candidate.

As shown in Fig. 7, PKS 2356-611 is located near one of the two ${ }^{55} \mathrm{Fe}$ calibration sources of the MECS instrument. In this case a careful choice of the background region is required 


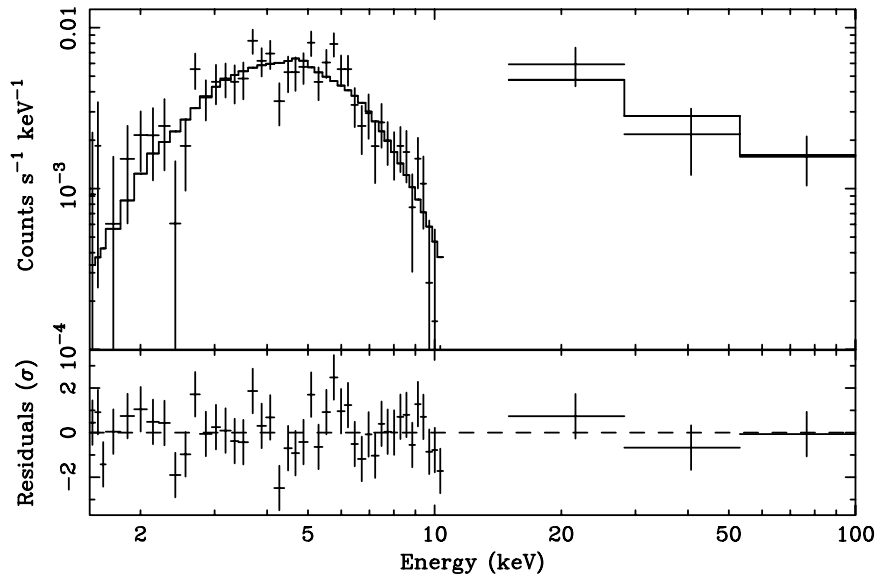

Fig. 8. Broad band spectrum of PKS 2356-611 fitted with an absorbed power law (upper panel) and residuals to this model in units of $\sigma$ (lower panel).

to avoid contamination by $5.95 \mathrm{keV}$ photons produced by the calibration source: this is done taking the background region as near as possible to the calibration sources itself.

Fitting the MECS data of PKS 2356-611 and the PDS points with a single power law plus intrinsic absorption provides an acceptable fit $\left(\chi^{2} / v=60 / 43\right)$, a steep spectrum ( $\Gamma=1.75$ ) and a column density of $N_{\mathrm{H}} \sim 1 \times 10^{23} \mathrm{~cm}^{-2}$ (Fig. 8). Both spectral parameters are typical of Seyfert 2 galaxies, and seem to confirm the source classification made by Lipovetsky et al. (1988).

The cross-calibration constant in this case turns out to be lower $(0.2-0.5)$ than generally observed. This could be due to imperfect background correction in the MECS because of the location of the source near the calibrator. However, the spectral parameters do not change significantly when this constant is constrained to vary within the nominal range of values.

The broad band spectrum of PKS 2356-611 (see Fig. 8) suggests the possible presence of an excess around $\sim 6 \mathrm{keV}$. Although the addition of an extra component in the form of a narrow Gaussian line at around $6.4 \mathrm{keV}$ with $E W \sim 500 \mathrm{eV}$ provides an improvement in the fit $\left(\Delta \chi^{2}=11.5\right.$ for two additional parameters), its reality is questioned by the location of the source near the ${ }^{55} \mathrm{Fe}$ calibrator: residuals at this line energy could still be due to a non-perfect subtraction of the calibration line. Also in this case the cross-calibration constant is low $(<0.5)$ and the $\chi^{2}$ values as well as the spectral parameters do not change significantly if we vary the cross-calibration constant within the allowed range. The absorption measured would produce an iron line of 100-200 eV in transmission, while reflection in the torus would increase the $E W$ to about $400 \mathrm{eV}$ (Turner et al. 1997) possibly indicating the presence of a reflection component. However, inclusion in the fit of this component via the PEXRAV model in XSPEC (Magdziarz \& Zdziarski 1995) is not required by the data and does not improve the cross-calibration constant, which turns out to be very small $(<0.4)$. A large $E W$ is also possible if the source is Compton thick; this type of object is generally characterized by a low $F_{\mathrm{X}} / F_{\text {OIII }}$ ratio (Bassani et al. 1999). In PKS $2356-611$ this ratio is $\sim 67$, when $F_{\text {OIII }}$ is corrected for the reddening in the galaxy using the observed value of $\mathrm{H}_{\alpha} / \mathrm{H}_{\beta}=4.32$ (Koekemoer et al. 1998). This result suggests that PKS 2356-611 is a Compton thin Seyfert 2 galaxy rather than a Compton thick one and so the large $E W$ is probably not due to strong absorption $\left(N_{\mathrm{H}}>1.5 \times 10^{24} \mathrm{~cm}^{-2}\right)$.

\subsection{Sources not detected in the MECS field of view}

In this section we describe the remaining eight fields where the source responsible for the PDS spectrum is not observed by the MECS. In Table 5 we report for each of these fields the source/s observed in the MECS, their flux extrapolated to the $20-100 \mathrm{keV}$ band and their contribution to the PDS flux. In Table 6 instead we list for each field the PDS exposure and count rate as well as the photon index $\Gamma$ and observed 20-100 keV flux obtained assuming a simple power law fit. We also report the effective flux at the source, estimated from that observed by applying the correction factor $R$.

\subsubsection{The IRAS $01025-6423$ field}

In this field the target source, IRAS 01025-6423, a Seyfert 2 galaxy, is barely detected by MECS at a $\sim 2 \sigma$ level, while the LECS data are not available. Due to the low signal to noise ratio, we only attempted to put an upper limit on the flux assuming a power law model with photon index $\Gamma=2$. The source appears to be faint in the $2-10 \mathrm{keV}$ range with a flux $\leq 3.1 \times 10^{-14} \mathrm{erg} \mathrm{cm}^{-2} \mathrm{~s}^{-1}$ and its contribution to the PDS signal is obviously null (see Table 5). Searching in the HEASARC archive for possible contaminating sources within the PDS field of view, we found two possible candidates belonging to the ROSAT Bright Source Catalog: 1RXS J011316.2-641142 (CPD-64 120), classified as a K1 Ve star, and 1RXS J010333.7-643925 (PKS 0101-649), identified as a radio source, at distances from the target source of $\sim 58^{\prime}$ and $\sim 33^{\prime}$, respectively. PKS 0101-649, which is the closest to the target source, is more likely to be the object responsible for the high energy emission, as a star is not expected to emit above $10 \mathrm{keV}$. PKS 0101-649 belongs to the 2 Micron All Sky Survey eXtended object (i.e. 2MASX J01033376-6439079 in NED), with a total photometry of $J=15, H=14$ and $K=13.4$ mag; the USNO-A2 R mag is 16.5 (Monet et al. 1999) thus providing an $R-K$ value of 3.1 which suggests a quite red object; at radio frequencies it is fairly bright with a $6 \mathrm{~cm}$ flux of $\sim 270 \mathrm{mJy}$.

The PDS spectrum is characterized by a photon index $\Gamma \sim 1.5$ (see Table 6) compatible, within uncertainties, with an AGN spectrum; the $20-100 \mathrm{keV}$ flux is $2.3 \times 10^{-11} \mathrm{erg} \mathrm{cm}^{-2} \mathrm{~s}^{-1}$, making PKS 0101-649 a bright source at these energies. If we extrapolate this power law to soft $\mathrm{X}$-ray energies, we find a $0.1-2.4 \mathrm{keV}$ flux of $0.22 \times 10^{-11} \mathrm{erg} \mathrm{cm}^{-2} \mathrm{~s}^{-1}$ which is consistent with the ROSAT value of PKS 0101-649. Alternatively, the X-ray source spectrum could be steeper but highly absorbed even above $10 \mathrm{keV}$ as observed in Compton thick objects (Matt et al. 2000b). If we add absorption to the power law, fixing the photon index to 1.9 , we find an upper limit to the column density $<2 \times 10^{25} \mathrm{~cm}^{-2}$. Obviously, an optical spectrum 
Table 6. PDS spectral analysis of serendipitous sources not detected in the MECS instrument.

\begin{tabular}{|c|c|c|c|c|c|c|c|}
\hline Source & $\begin{array}{l}\text { Offset }^{(a)} \\
(\operatorname{arcmin})\end{array}$ & $\begin{array}{l}\text { EXPO } \\
(\mathrm{ks})\end{array}$ & $\mathrm{cts} / \mathrm{s}$ & $\Gamma$ & $\begin{array}{l}R^{(b)} \\
(\%)\end{array}$ & $\begin{array}{c}F_{\text {Obs }}^{(c)} \\
{[20-100] \mathrm{keV}}\end{array}$ & $\begin{array}{c}F_{\text {Source }}^{(c)} \\
{[20-100] \mathrm{keV}}\end{array}$ \\
\hline PKS 0101-649 & 32.5 & 26.4 & $0.115 \pm 0.035$ & $1.51_{-1.03}^{+1.45}$ & 56 & 12.8 & 23.0 \\
\hline Perseus/NGC 1275 (?) & 62.0 & 25.9 & $0.438 \pm 0.043$ & $3.27_{-0.43}^{+0.53}$ & 19 & 25.0 & 136.0 \\
\hline NGC $1566^{(d)}$ & 60.2 & 26.6 & $0.192 \pm 0.041$ & $2.50_{-0.84}^{+1.32}$ & 19 & 15.4 & 81.1 \\
\hline NGC $3227^{(d)}$ & 55.1 & 176.0 & $0.247 \pm 0.016$ & $1.85_{-0.22}^{+0.24}$ & 26 & 25.5 & 98.1 \\
\hline ESO 025-G002 & 31.4 & 26.1 & $0.138 \pm 0.035$ & $2.96_{-0.93}^{+0.22}$ & 55 & 11.1 & 20.2 \\
\hline $4 \mathrm{C}+74.26^{(d)}$ & 34.2 & 66.2 & $0.250 \pm 0.024$ & $2.03_{-0.31}^{+0.37}$ & 53 & 24.4 & 46.0 \\
\hline NGC 7319 & 33.7 & 29.2 & $0.137 \pm 0.031$ & $2.07_{-0.53}^{+0.67}$ & 59 & 14.1 & 24.0 \\
\hline NGC 7582 & 27.6 & 56.8 & $0.490 \pm 0.027$ & $1.73_{-0.16}^{+0.18}$ & 61 & 49.6 & 81.3 \\
\hline
\end{tabular}

Note: Count rates are referred to the energy bands used in the spectral analysis (see text).

(a) Distance between the pointing target and the off-axis sources.

(b) Source flux correction factor due to the PDS response to sources not on-axis.

(c) In units of $10^{-12} \mathrm{erg} \mathrm{cm}^{-2} \mathrm{~s}^{-1}$.

${ }^{(d)}$ For this source the parameters of the average spectrum are reported.

would be highly desirable to provide more information and establish the true nature of this source.

\subsubsection{The MKN 1073 field}

The Seyfert 2 galaxy MKN 1073 was not clearly detected by the LECS and the MECS instruments. In both detections there is evidence for diffuse emission probably associated with the Perseus cluster, which is at $62^{\prime}$ from the centre of the MECS pointing. The signal in the PDS is high $(>10 \sigma$ level $)$, with an exceptionally steep spectrum $(\Gamma \sim 3.3$, see Table 6$)$. We ascribe the PDS emission to the Perseus cluster, which has also been targetted by BeppoSAX on September 1996. The spectral analysis of these PDS data also shows a very steep spectrum $(\Gamma=$ $3.80_{-0.18}^{+0.17}$ ) and a $20-100 \mathrm{keV}$ flux of $\sim 5 \times 10^{-11} \mathrm{erg} \mathrm{cm}^{-2} \mathrm{~s}^{-1}$, lower than the flux seen during the MKN 1073 observation (see Table 6). Since flux variability in the cluster is unlikely, the change in the emission could be due to the Seyfert 2 galaxy NGC 1275, often taken as responsible for the high energy (>20 keV) emission observed from this region; comparison of data from high energy instruments over years shows a large variation in the flux above $20 \mathrm{keV}$ (Osako et al. 1994). It is also possible that both cluster and AGN contribute to the high energy emission, but it is difficult at present to disentangle one contribution from the other.

\subsubsection{The NGC 1553 field}

Chandra observations of the X-ray faint SO galaxy NGC 1553 (Blanton et al. 2001) has spatially and spectrally resolved the source of the X-ray emission. A significant fraction of this $(\sim 70 \%)$ is detected as diffuse flux while the remainder is due to 49 objects. The strongest source in the field is located at the centre of NGC 1553 to within 0.5 and shows a hard spectrum, typical of an AGN. NGC 1553 was observed twice by BeppoSAX (January 1997 and November 1997). Trinchieri et al. (2000) performed the spectral analysis of the LECS and MECS data finding as best-fit a RAYMONDSMITH model $(k T \simeq 0.26 \mathrm{keV})$ for the soft component and a thermal bremsstrahlung ( $k T \simeq 4.8 \mathrm{keV}$ ) for the hard component. Our analysis of the LECS and MECS data from both observations is in agreement with that reported by these authors; the extrapolation of this model to high energies provides a very low contribution to the PDS flux (see Table 5). Searching in past X-ray mission archives, we find that the Seyfert 1 galaxy NGC 1566 is the best candidate for the PDS emission as also suggested by Trinchieri et al. (2000). This source is at a distance of 60.2 from the target object and is therefore just inside the PDS field of view. NGC $1566(z=0.005, B=9.9$, $R=9.6$ ) is a very bright $\mathrm{X}$-ray source known since the HEAO 1 X-ray Source Catalog (Wood et al. 1984). The source belongs also to the 2 Micron All Sky Survey eXtended Source Catalog (2MASX J04200041-5456161), with magnitudes $J=7.8$, $B=7.2$ and $K=6.9$, it is reported by ROSAT in the Bright Source Catalog and it is a radio source of $\sim 100 \mathrm{mJy}$ at $6 \mathrm{~cm}$. The $2-10 \mathrm{keV}$ flux reported in the literature ranges from $<0.5$ to $1.7 \times 10^{-11} \mathrm{erg} \mathrm{cm}^{-2} \mathrm{~s}^{-1}$ (Halpern 1982) indicating variability by around a factor of 3 . The PDS emission shows a variation of $\sim 70 \%$ during our two observations, but due to the poor statistics in both measurements (the signal to noise ratio in the PDS is around $3 \sigma$ level in each measurement), we summed the observations to improve the statistics and performed an averaged spectral analysis. As shown in Table 6, this analysis indicates a photon index $\Gamma$ of $\sim 2.5$ and an average $20-100 \mathrm{keV}$ flux, corrected for the offset, of $\sim 8 \times 10^{-11} \mathrm{erg} \mathrm{cm}^{-2} \mathrm{~s}^{-1}$. By extrapolating the flux down to the $2-10 \mathrm{keV}$ range, we find a value of $\sim 2 \times 10^{-10} \mathrm{erg} \mathrm{cm}^{-2} \mathrm{~s}^{-1}$, much higher with respect to previous measurements; however, if the photon index is restricted to values more appropriate for a Seyfert 1 galaxy (i.e. 1.7-1.9), then the $2-10 \mathrm{keV}$ flux reduces to $\sim 6 \times 10^{-11} \mathrm{erg} \mathrm{cm}^{-2} \mathrm{~s}^{-1}$, only a factor of 3 higher. This result indicates that NGC 1566 could indeed dominate the PDS emission and that flux variability is likely.

\subsubsection{The AD Leonis field}

The M-dwarf Ad Leonis was the subject of an observational campaign performed by BeppoSAX (April 1997, 1, 8 
and 12 May 1999). We adopt the multi-temperature model (MEKAL code in XSPEC, Mewe et al. 1985) proposed by van den Besselaar et al. (2003) for the XMM-Newton and Chandra observations, and find that the LECS and MECS data of the four observations are best described by a two-temperature model, in which the metal abundances are left free to vary. The $2-10 \mathrm{keV}$ flux varies in the range $1.4-2.3 \times$ $10^{-12} \mathrm{erg} \mathrm{cm}^{-2} \mathrm{~s}^{-1}$, corresponding to a flux variation of $\sim 60 \%$, while the spectral parameters do not show significant variation. In view of these indications, we summed the data from different pointings and estimated the average spectral parameters. The two-temperature model remains the best fit $\left(\chi^{2} / v=97 / 82\right)$, giving $k T_{1}=0.65_{-0.02}^{+0.03} \mathrm{keV}$ and $k T_{2}=2.04_{-0.17}^{+0.24} \mathrm{keV}$ metal abundances of $0.14 \pm 0.02$ and $0.56_{-0.21}^{+0.25}$ respectively and an averaged 2-10 keV flux of $\sim 2 \times 10^{-12} \mathrm{erg} \mathrm{cm}^{-2} \mathrm{~s}^{-1}$; this spectrum if extrapolated to high energies provides almost no contribution to the PDS flux (see Table 5).

At a distance of $\sim 55^{\prime}$ from AD Leonis, we find the Seyfert 1 galaxy NGC 3227, which could be the contaminating source we are searching for. NGC 3227, first detected in the Ariel $V$ all sky survey, is one of the bright sources in the Piccinotti sample of AGN (Piccinotti et al. 1982). NGC 3227 is known to be variable (on time scales of hours-days) and characterized by a spectrum with a photon index $\Gamma \sim 1.6$, flatter than that typically observed in Seyfert galaxies (George et al. 1998). Ginga observations performed in 1988 indicate a 2-10 keV flux of $\sim 4 \times 10^{-11} \mathrm{erg} \mathrm{cm}^{-2} \mathrm{~s}^{-1}$ (Pounds et al. 1989), while ASCA observations performed during 1993 and 1995 provide $2-10 \mathrm{keV}$ flux measurements in the range $2.4-2.6 \times 10^{-11} \mathrm{erg} \mathrm{cm}^{-2} \mathrm{~s}^{-1}$. Recently, Lamer et al. (2003), analyzing the Rossi X-ray Timing Explorer (RXTE) data of NGC 3227, confirmed the source variability, explaining it in terms of transient absorption by a gas cloud (neutral or weakly ionized) of column density of $\sim 3 \times$ $10^{23} \mathrm{~cm}^{-2}$ moving across the line of sight to the X-ray source. This interpretation has been confirmed also by XMM-Newton observations (Gondoin et al. 2003). NGC 3227 has been detected at higher energies by OSSE and BATSE on-board the Compton Gamma Ray Observatory (CGRO). The OSSE data when fitted in the 50-200 keV range, show a spectral index $\Gamma=1.86_{-0.38}^{+0.36}$, softer with respect to that found in the $2-10 \mathrm{keV}$ band and a photon flux of $(3.40 \pm 0.55) \times 10^{-4} \mathrm{~cm}^{-2} \mathrm{~s}^{-1}$ in the $50-150 \mathrm{keV}$ band (Zdziarski et al. 2000). Based on these measurements, we estimate a $20-100 \mathrm{keV}$ flux measurement in the range $5.0-7.8 \times 10^{-11} \mathrm{erg} \mathrm{cm}^{-2} \mathrm{~s}^{-1}$. In addition, the BATSE data (Malizia et al. 1999) show a $20-100 \mathrm{keV}$ flux in the range $9.7-12.8 \times 10^{-11} \mathrm{erg} \mathrm{cm}^{-2} \mathrm{~s}^{-1}$. The spectral analysis of our PDS data indicate that, during the four observations of AD Leonis, the 20-100 keV flux measurements of NGC 3227 ranged in the interval $6.3-14.7 \times 10^{-11} \mathrm{erg} \mathrm{cm}^{-2} \mathrm{~s}^{-1}$, with an averaged value of $9.8 \times 10^{-11} \mathrm{erg} \mathrm{cm}^{-2} \mathrm{~s}^{-1}$ and a photon index $\Gamma \sim 1.85$ (see Table 6 ). These results turn out to be in perfect agreement with both OSSE and BATSE data. We want to stress that our measurement contains the only spectral information about NGC 3227 obtained by BeppoSAX (the source has never been observed by the satellite) and so represent an interesting addition to the list of Piccinotti sample sources for which BeppoSAX high energy data are available.

\subsubsection{The $\mathrm{H} 1846-786$ field}

H1846-786 is a Seyfert 1 galaxy belonging to the Piccinotti sample (Piccinotti et al. 1982). Despite its X-ray brightness, the source has been poorly studied. A simple power law, with a photon index $\Gamma \sim 1.95$ is the best-fit model to the LECS and MECS data $\left(\chi^{2} / v=125 / 100\right)$ as obtained by Quadrelli et al. (2003). This result indicates that no extra absorption and/or an iron line are present in the spectrum. The extrapolation of this best-fit model to the PDS energy range shows a significant contribution from H1846-786 (see Table 5). In fact, as can be seen in Table 1, this case represents the lowest range for the PDS/MECS normalization constant, indicating that both the target (H1846-786) and another object, located within the PDS field of view, contribute to the PDS flux. This excess emission could be due to the near $(z=0.0285, B=8.8$, $R=10.4$ ) bright Seyfert 1 galaxy ESO 025-G002, which has a ROSAT flux only 5 times lower than the target source. Also this source belongs to the 2 Micron All Sky Survey eXtended Source Catalog (i.e. 2MASX J18544039-7853544), with magnitudes $J=11.4, H=10.6$ and $K=10.3$, and shows weak radio emission $(\sim 10 \mathrm{mJy}$ at $36 \mathrm{~cm})$. The spectral analysis of the PDS data yields a contribution from this object of $\sim 60 \%$. The spectrum is steep $(\Gamma \sim 3)$ but still compatible with the canonical AGN value of 2 and the $20-100 \mathrm{keV}$ flux turns out to be of the order of $2.0 \times 10^{-11} \mathrm{erg} \mathrm{cm}^{-2} \mathrm{~s}^{-1}$ (see Table 6 for more details). Thus, ESO 025-G002 shows at high energies a flux of the same order of that of H1846-786, while at soft energies is 5 time lower. This can be explained if the source is absorbed at soft energies; in fact, fixing the PDS power law index to a value more appropriate to an AGN and allowing for absorption, we find an upper limit to the column density of a few $10^{22} \mathrm{~cm}^{-2}$.

Finally, we can conclude that in this particular case both target and serendipitous source are likely to give roughly the same contribution to the PDS emission.

\subsubsection{The VW Cephei field}

VW Cephei, a W UMa-type binary system, has been observed by BeppoSAX twice (May 1998 and October 1998). Following the investigations performed by Gondoin (2004), we fit separately the LECS and MECS data of both data sets with the MEKAL optically thin plasma model. In each observation, a three-component model, having different temperatures but the same metal abundance, gives the best representation of the data. The $2-10 \mathrm{keV}$ flux turns out to be $1.6 \times 10^{-12} \mathrm{erg} \mathrm{cm}^{-2} \mathrm{~s}^{-1}$ and $0.8 \times 10^{-12} \mathrm{erg} \mathrm{cm}^{-2} \mathrm{~s}^{-1}$ in the first and second pointings, respectively. Although the flux varies $(\sim 50 \%)$ from the first to the second observation, the spectral shape shows, within the uncertainties, no evidence of variability; therefore, we summed the two data sets. The three-temperature model applied to the combined data still provides a good fit $\left(\chi^{2} / v=37 / 65\right)$ and gives temperature values of 2.4, 0.69 and $0.13 \mathrm{keV}$; extrapolation of this model to the PDS band indicates that the VW Cephei contribution to the PDS data is negligible (see Table 5).

As a possible contaminating source, we find at around $34^{\prime}$ from VW Cephei an object identified with $4 \mathrm{C}+74.26$, a radio-loud active galaxy. This is a particularly interesting 
object because its ASCA X-ray spectrum (Brinkmann et al. 1998; Sambruna et al. 1999) shows features that are typical of Seyfert galaxies more than of Broad Line Radio Galaxies: a warm absorber and a significant Compton reflection hump. It is also the only quasar in the collection of Sambruna et al. (1999) with a detectable $\mathrm{Fe}_{\mathrm{K} \alpha}$ line. 4C +74.26 was targetted by BeppoSAX on May 1999. Analysis of these data by Hasenkopf et al. (2002) indicated as the best-fit model a power law continuum modified by Compton reflection at high energies and by absorption at low energies. An iron line was also detected, but its energy could not be tightly constrained, falling between 6.4 and $6.9 \mathrm{keV}$. Our spectral analysis of the average PDS emission obtained by combining the two observations of VW Cephei yields a photon index $\Gamma \sim 2$ and a $20-100 \mathrm{keV}$ flux, corrected for the off-axis effect, of $4.6 \times 10^{-11} \mathrm{erg} \mathrm{cm}^{-2} \mathrm{~s}^{-1}$ (see Table 6). If we fit the PDS spectrum with the PEXRAV model, fixing the inclination angle to the value indicated by Hasenkopf et al. (2002), we find a satisfactory fit $\left(\chi^{2} / v=1.2 / 2\right)$, but the reflection coefficient of $R \sim 1.3$ and the cut-off energy $E_{\mathrm{C}}>148 \mathrm{keV}$ are not well constrained, although in agreement with what reported by Hasenkopf et al. (2002). In order to check the self-consistency of our spectral analysis, we reanalysed the PDS data of the May 1999 observation with a simple power law and obtained spectral parameters for this epoch (a photon index $\Gamma \sim 2$ and a 20-100 keV flux of $\left.4.0 \times 10^{-11} \mathrm{erg} \mathrm{cm}^{-2} \mathrm{~s}^{-1}\right)$ consistent with those obtained during the VW Cephei pointings.

Overall, the data indicate that $4 \mathrm{C}+74.26$ is the most likely contaminating source present in the PDS field of view of VW Cephei and further suggest no variability in the high energy flux.

\subsubsection{The NGC 7331 field}

A good fit to the MECS and LECS data of the LINER NGC 7331 is provided by a single power law having a photon index $\Gamma=1.95_{-0.18}^{+0.12}$ and $2-10 \mathrm{keV}$ flux of $5.1 \times$ $10^{-13} \mathrm{erg} \mathrm{cm}^{-2} \mathrm{~s}^{-1}$. This result confirms the non-thermal X-ray spectrum of NGC 7331 reported by Stockdale et al. (1998) on the basis of both ROSAT and radio observations. The above model however gives a small contribution (see Table 5) to the PDS emission when extrapolated to high energies.

Searching for potential high energy emitters in the HEASARC archives, we find a source located at about $30^{\prime}$ from the centre of the MECS pointing and identified with the Seyfert 2 galaxy NGC 7319, which belongs to the Stephan's Quintet, a compact group of galaxies. Recent Chandra data of NGC 7319 (Trinchieri et al. 2003) describe the source emission as due to the superposition of a strong and heavy absorbed nuclear source plus diffuse softer emission. Modeling the nuclear source with a combination of absorbed and unabsorbed power law components having the same photon index, plus a narrow $6.4 \mathrm{keV}$ emission line, the above authors found a photon index $\Gamma \sim 1.7$, a column density $N_{\mathrm{H}}=4 \times 10^{23} \mathrm{~cm}^{-2}$, an $E W \sim 110 \mathrm{eV}$ and a $2-10 \mathrm{keV}$ flux of $8.2 \times 10^{-12} \mathrm{erg} \mathrm{cm}^{-2} \mathrm{~s}^{-1}$. The spectral fit was similar to that derived from a previous ASCA observation (Awaki et al. 1997), except for the smaller $E W$ and higher 2-10 keV flux. The X-ray spectral parameters

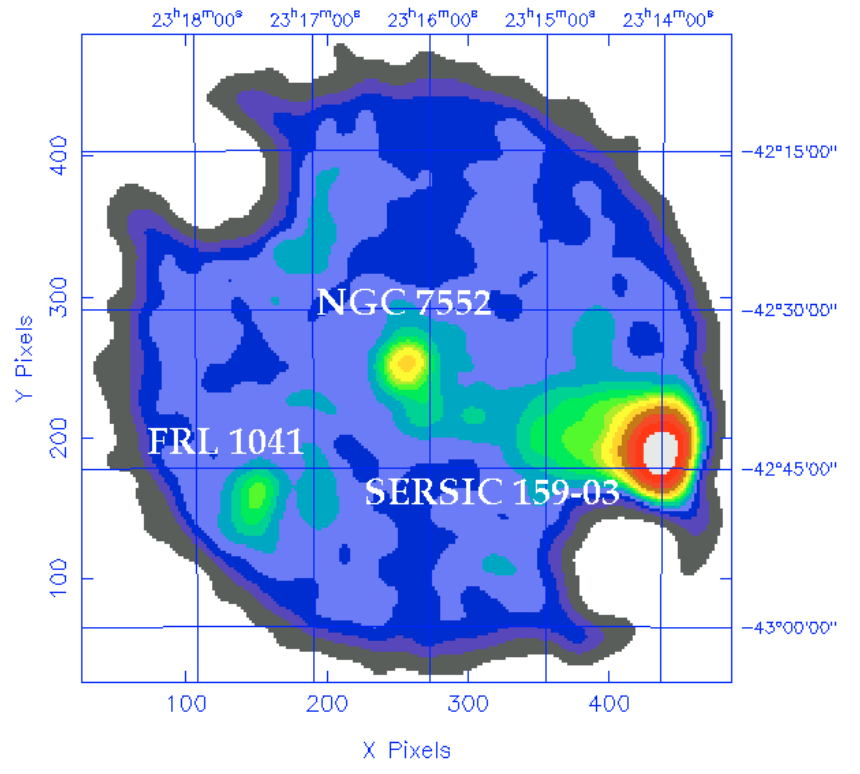

Fig. 9. MECS image of the sky region surrounding NGC 7552.

as well as the $F_{\mathrm{X}} / F_{\mathrm{OIII}}$ ratio (Bassani et al. 1999) indicate that the source is likely to be Compton thin. NGC $7319(z=0.0225$, $B=14, R=12.7$ ) belongs to the 2 Micron All Sky Survey eXtended Source Catalog (2MASX J22360355+3358327), with magnitudes $J=11.1, H=10.3$ and $K=10.1$, and is also detected in radio $(\sim 7 \mathrm{mJy}$ at $6 \mathrm{~cm})$.

The PDS data are well described by a power law with a photon index $\Gamma \sim 2.1$ (see Table 6) and a 20-100 keV flux of $2.4 \times 10^{-11} \mathrm{erg} \mathrm{cm}^{-2} \mathrm{~s}^{-1}$ at the source. By extrapolating the PDS spectrum to the $2-10 \mathrm{keV}$ band, we find a flux of $1.7 \times 10^{-11} \mathrm{erg} \mathrm{cm}^{-2} \mathrm{~s}^{-1}$, which is higher by a factor of $\sim 9$ and $\sim 2$ with respect to the ASCA and Chandra measurements, respectively. However, if we fit the PDS spectrum with an absorbed power law, fixing the column density $N_{\mathrm{H}}$ and the photon index $\Gamma$ to the best-fit values found by Trinchieri et al. (2003) and extrapolate to the $2-10 \mathrm{keV}$ energy range, we find a $2-10 \mathrm{keV}$ flux of $\sim 7 \times 10^{-12} \mathrm{erg} \mathrm{cm}^{-2} \mathrm{~s}^{-1}$ slightly lower than the Chandra value, but still compatible with it. This result strongly indicates that NGC 7319 is a good candidate to explain the PDS emission in the NGC 7331 observation.

\subsubsection{The NGC 7552 field}

In this field, although two extra sources are present within the MECS image (see Fig. 9), neither seems to be responsible for the high energy emission seen in the PDS.

The LECS and MECS data of the starburst galaxy NGC 7552 are well described by a two-component model, consisting of a thermal part (RAYMOND-SMITH model in XSPEC) with $k T=0.92_{-0.34}^{+0.28} \mathrm{keV}$ and a power law with a steep spectrum $\left(\Gamma=2.74_{-0.75}^{+0.91}\right)$. The $2-10 \mathrm{keV}$ flux is $1.6 \times 10^{-13} \mathrm{erg} \mathrm{cm}^{-2} \mathrm{~s}^{-1}$, and the extrapolation of this model to high energies shows an almost null $(<1 \%)$ contribution to the PDS emission.

As displayed in Fig. 9, within the MECS field of view we find two other sources. The first, located at $\alpha(2000)=$ $23^{\mathrm{h}} 17^{\mathrm{m}} 28^{\mathrm{s}} .8$ and $\delta(2000)=-42^{\circ} 47^{\prime} 36^{\prime \prime} .2$ is associated to the 
Seyfert 1 galaxy FRL 1041, while the second at $\alpha(2000)=$ $23^{\mathrm{h}} 14^{\mathrm{m}} 00^{\mathrm{s}} .7$ and $\delta(2000)=-42^{\circ} 43^{\prime} 17^{\prime \prime} \cdot 3$ is identified with the cluster of galaxies Sersic 159-03. Fitting the MECS data of FRL 1041 with a simple power law, provides a satisfactory fit $\left(\chi^{2} / v=14.9 / 15\right)$, a photon index $\Gamma=1.63_{-0.60}^{+0.67}$, compatible with values found for Seyfert galaxies, and a $2-$ $10 \mathrm{keV}$ flux of $5.0 \times 10^{-13} \mathrm{erg} \mathrm{cm}^{-2} \mathrm{~s}^{-1}$. The extrapolation of this model to high energies gives a low (see Table 5) contribution and a joint fit of the MECS/PDS data gives a crosscalibration constant (37-82) well outside the nominal range. Concerning the galaxy cluster, Sersic 159-03, we find that the best-fit model $\left(\chi^{2} / v=26.4 / 32\right)$, for the MECS data only, is a thermal bremsstrahlung having a temperature value $(k T=$ $2.54_{-0.22}^{+0.24} \mathrm{keV}$ ), compatible with that found by XMM-Newton observations (Kaastra et al. 2001), plus a narrow Gaussian line centered at $6.47_{-0.21}^{+0.24} \mathrm{keV}$ with an $E W$ of $552_{-327}^{+229} \mathrm{eV}$. The 2$10 \mathrm{keV}$ flux is $7.0 \times 10^{-12} \mathrm{erg} \mathrm{cm}^{-2} \mathrm{~s}^{-1}$. The quality of the fit is rather worse when we fit simultaneously the MECS and PDS data; in fact, we cannot put any constraint on the spectral parameters and, in addition, the PDS/MECS cross-calibration constant varies within the interval 91-303. The above results indicate that neither source is likely to be responsible for the high energy emission (see also Table 5), even in the case of the cluster which is strongly detected in the MECS instrument ( $\sim 50 \sigma$ level), but has an extremely soft spectrum.

In view of these findings, we performed a further search in order to understand if there was any other nearby object which could produce the PDS result. Just outside the MECS field of view we find NGC 7582, a Seyfert 2 galaxy, belonging to the Piccinotti sample, i.e. a very bright source in hard X-rays (Piccinotti et al. 1982; Malizia et al. 1999).

NGC 7582 has already been observed by BeppoSAX in November 1998 (Turner et al. 2000). In that case the broad spectrum $(2-100 \mathrm{keV})$ was described by a power law of photon index $\Gamma=1.95_{-0.18}^{+0.09}$ (steeper than the index found during a previous ASCA observation) transmitted through a dual absorber with column densities $N_{\mathrm{H}} \sim 1.6 \times 10^{24} \mathrm{~cm}^{-2}$ (covering $60 \%$ of the nucleus) and $\sim 1.44 \times 10^{23} \mathrm{~cm}^{-2}$ (fully covering the source). The authors reported a $10-100 \mathrm{keV}$ flux of $\sim 1.2 \times 10^{-10} \mathrm{erg}$ $\mathrm{cm}^{-2} \mathrm{~s}^{-1}$ and the presence of variability on time-scales down to a few thousand of seconds. In addition, Turner et al. (2000) found that NGC 7582 was significantly brighter than the average level sampled by OSSE $\left(\sim 4 \times 10^{-11} \mathrm{erg} \mathrm{cm}^{-2} \mathrm{~s}^{-1}\right.$ in the $50-150 \mathrm{keV}$ band $)$ and BATSE $\left(\sim 8.9 \times 10^{-11} \mathrm{erg} \mathrm{cm}^{-2} \mathrm{~s}^{-1}\right.$ in the 20-100 keV band) (Johnson et al. 1997; Malizia et al. 1999), both estimated using a power law model.

By subtracting from the PDS the contribution due to each of the three sources present in the MECS image of Fig. 9 and fitting the remaining emission with a simple power law, we find a photon index of 1.72 (see Table 6) and a $20-100 \mathrm{keV}$ flux $(50-150 \mathrm{keV})$ at the source of $8.13 \times 10^{-11} \mathrm{erg} \mathrm{cm}^{-2} \mathrm{~s}^{-1}$ $\left(\sim 5 \times 10^{-11} \mathrm{erg} \mathrm{cm}^{-2} \mathrm{~s}^{-1}\right)$, which is in perfect agreement with the values reported by BATSE and OSSE. If we fit our data, taking into account the double absorber proposed by Turner et al. (2000), we find a photon index $\Gamma \sim 1.9$ and a $10-100 \mathrm{keV}$ flux of $2.3 \times 10^{-10} \mathrm{erg} \mathrm{cm}^{-2} \mathrm{~s}^{-1}$, a factor of 2 higher than the value reported for the 1998 observation by Turner et al. (2000).
To conclude, NGC 7582 is very likely the serendipitous source responsible for the emission detected above $10 \mathrm{keV}$ in the pointing of NGC 7552. Furthermore, we provide evidence for variability $(\sim 90 \%)$ in the flux between the two BeppoSAX measurements taken one year apart.

\section{Conclusions}

In this work we have shown how a careful search in the BeppoSAX public archive can provide evidence for new hard X-ray emitting sources, most likely associated with active galaxies, and/or confirm the high energy emission of known objects. In particular, we report the detection of six new hard $\mathrm{X}$-ray emitters (two type 1 Seyfert galaxies, two type 2, one quasar and a galaxy not yet classified as active); for two of these sources broad band spectra are presented, while in four cases only results above $10 \mathrm{keV}$ are reported.

For the remaining 6 objects in our sample, emission above $10 \mathrm{keV}$ was known before this work, mainly from previous dedicated BeppoSAX pointings. In the case of NGC 3227, an object known to emit at high energies from past OSSE and BATSE measurements, a spectrum is published here for the first time. Comparison of the present data with previous observations either with BeppoSAX and/or with other high energy missions indicates that flux variability is present in three (MKN 766, NGC 7582 and NGC 3227) possibly four (NGC 1275) objects.

As the AGN reported in this paper are "loosely" representative of the population of extragalactic objects with emission above $10 \mathrm{keV}$, it is worth examining their overall characteristics. The first consideration to make is that most of our objects ( 6 out of 10 with optical classification) are broad line emitting or unabsorbed AGN, contrary to the expectation of finding more absorbed than unabsorbed objects. The other four (possibly five if we include PKS 0101-649) show intrinsic absorption which, however, is compatible with a Compton thin nature $\left(N_{\mathrm{H}}<10^{24} \mathrm{~cm}^{-2}\right)$. Only in the case of PKS 0101-649 could the source be Compton thick. This is probably due to the limited capability of current hard X-ray detectors which allow just the brightest and nearest extragalactic objects to be found. In fact, all but two (Perseus and 4C +74.26 ) or even one (if NGC 1275 is responsible for the emission detected by the PDS) of our AGNs are nearby $(z<0.1)$ and belong to the 2 Micron All Sky Survey eXtended Source Catalog (i.e. the surrounding galaxy is visible in the near-infrared). At least eight (possibly nine if we also consider NGC 1275) of our objects are radio loud, according to the Terashima \& Wilson (2003) definition.

Overall, we can conclude that our objects are bright, nearby, with a detectable emission in all wavebands and only a fraction $(\sim 30-40 \%)$ have a column density in excess of $10^{22} \mathrm{~cm}^{-2}$

As shown here it is also possible to encounter offset fields contaminated by the presence of serendipitous sources. We are now performing a systematic analysis of all PDS data present in the archive (above $15^{\circ}$ in galactic latitudes) evaluating the relative offset fields; the aim of this work is to provide a list of positive detections in these background measurements. We are confident that this analysis will provide new high energy sources as highlighted here in three cases. 
Acknowledgements. This research has made use of SAXDAS linearized and cleaned event files of LECS and MECS produced at ASI Science Data Center; and of the High Energy Science Archive Research Center (HEASARC), provided by NASA's Goddard Space Flight Center. We acknowledge the financial support of the Italian Space agency (ASI) through contract ASI/CNR I/R/073/02. We thank J.B. Stephen for a careful reading of the manuscript. We also thank the referee for useful comments and suggestions.

\section{References}

Arnaud, K. A. 1996, in Astronomical Data Analysis Software and Systems V, ed. G. H. Jacoby, \& J. Barnes (San Francisco: ASP), ASP Conf. Ser., 101, 17

Awaki, H., Koyama, K., Matsumoto, H., et al. 1997, PASJ, 49, 445

Bassani, L., Dadina, M., Maiolino, R., et al. 1999, ApJS, 121, 473

Bassani, L., Malizia, A., Stephen, J. B., et al. 2004, Proc. Fifth INTEGRAL Workshop, ESA SP-552

Bird, A. J., Barlow, E. J., Bassani, L., et al. 2004, ApJ, 607, L33

Blanton, E. L., Sarazin, C. L., \& Irwin, J. A. 2001, ApJ, 552, 106

Boella, G., Butler, R. C., Perola, G. C., et al. 1997a, A\&AS, 122, 299

Boella, G., Chiappetti, L., Conti, G., et al. 1997b, A\&AS, 122, 327

Bregman, J. N., \& Pildis, R. A. 1994, ApJ, 420, 570

Brinkmann, W., Otani, C., Wagner, S. J., \& Siebert, J. 1998, A\&A, 330,67

Chiappetti, L., \& Dal Fiume, D. 1997, in Data Analysis in Astronomy, ed. V. Di Gesu, M. J. B. Duff, A. Heck, et al. (Singapore: World Scientific), 111

Dermer, C. D., \& Gherels, N. 1995, ApJ, 447, 103

Dickey, J. M., \& Lockman, F. J. 1990, ARA\&A, 28, 215

Eracleous, M., Halpern, J. P., \& Livio, M. 1996, ApJ, 459, 89

Fabian, A. C. 2001, in X-Ray Astronomy 2000, ed. R. Giacconi, S. Serio, \& L. Stella (San Francisco: ASP), ASP Conf. Proc., 234, 545

Fiore, F., Guainazzi, M., \& Grandi, P. 1999a, SAXabc, vs. 1.2, Cookbook for BeppoSAX NFI Spectral Analysis

Fiore, F., La Franca, F., Giommi, P., et al. 1999b, MNRAS, 306, L55

Frontera, F., Cinti, M.N., Dal Fiume, D., et al. 1997a, Nuovo Cimento C, 20, 797

Frontera, F., Costa, E., Dal Fiume, D., et al. 1997b, A\&AS, 122, 357

Gehrels, N., Chincarini, G., Giommi, P., et al. 2004, ApJ, 611, 1005

George, I. M., Mushotzky, R., Turner, T. J., et al. 1998, ApJ, 509, 146

Gondoin, P. 2004, A\&A, 415, 1113

Gondoin, P., Orr, A., Lumb, D., \& Siddiqui, H. 2003, A\&A, 397, 883

Grandi, P., Guainazzi, M., Haardt, F., et al. 1999, A\&A, 343, 33

Halpern, J. P. 1982, Ph.D. Thesis Harvard University, Cambridge, MA

Hasenkopf, C. A., Sambruna, R. M., \& Eracleous, M. 2002, ApJ, 575, 127

Johnson, W. N., Zdziarski, A. A., Madejski, G. M., et al. 1997, in Proc. Fourth Compton Symp., Part 1, ed. C. D. Dermer, M. S. Strickman, \& J. D. Kurfess (New York: AIP), AIP Conf. Proc., 410, 283

Inda, M., Makishima, K., Kohmura, Y., et al. 1994, ApJ, 420, 143
Kaastra, J. S., Ferrigno, C., Tamura, T., et al. 2001, A\&A, 365, 99 Koekemoer, A. M., \& Bicknell, G. V. 1998, ApJ, 497, 662

Kruper, J. S., Canizares, C. R., \& Urry, C. M. 1990, ApJS, 74, 347

Lamer, G., Uttley, P., \& Hardy, I. M. M. 2003, MNRAS, 342, L41

Leighly, K. M., Mushotzky, R. F., Yaqoob, T., Kunieda, H., \& Edelson, R. 1996, ApJ, 469, 147

Leighly, K. M., O’Brien, P. T., Edelson, R., et al. 1997, ApJ, 483, 767

Levine, A. M., Lang, F. L., Lewin, W. H. G., et al. 1984, ApJS, 54, 581

Lipovetsky, V. A., Neitzvestny, S. I., \& Neitzvestnaya, O. M. 1988, Soobshch. Spets. Artrof. Obs., 55, 5

Loaring, N. S., Page, M. J., \& Ramsay, G. 2003, MNRAS, 345, 865

Magdziarz, P., \& Zdziarski, A. A. 1995, MNRAS, 273, 837

Malizia, A., Bassani, L., Zhang, S. N., et al. 1999, ApJ, 519, 637

Matt, G., Perola, G. C., Fiore, F., et al. 2000a, A\&A, 363, 863

Matt, G., Fabian, A. C., Guainazzi, M., et al. 2000b, MNRAS, 318, 173

Mewe, R., Gronenschild, E. H. B. M., \& van den Oord, G. H. J. 1985, A\&AS, 62, 197

Monet, A. K. B., Levine, S. E., Monet, D. G., et al. 1999, BAAS, 31, 1532

Monet, D. G., Levine, S. E., Canzian, B., et al. 2003, AJ, 125, 984

Nandra, K., \& Pounds, K. A. 1994, MNRAS, 268, 405

Osako, C. Y., Ulmer, M. P., Grabelsky, D. A., et al. 1994, ApJ, 435, 181

Pan, H. C., Jordan, C., Makishima, K., et al. 1997, MNRAS, 285, 735

Parmar, A. N., Martin, D. D. E., Bavdaz, M., et al. 1997, A\&AS, 122, 309

Perri, M., Massaro, E., Giommi, P., et al. 2003, A\&A, 407, 453

Piccinotti, G., Mushotzky, R. F., Boldt, E. A., et al. 1982, ApJ, 253, 485

Pounds, K. A., Nandra, K., Stewart, G. C., \& Leighly, K. 1989, MNRAS, 240, 769

Quadrelli, A., Malizia, A., Bassani, L., \& Malaguti, G. 2003, A\&A, 411, 77

Raymond, J. C., \& Smith, B. W. 1977, ApJS, 35, 419

Sambruna, R. M., Eracleous, M., \& Mushotzky, R. F. 1999, ApJ, 526, 60

Schwope, A. D., Hasinger, G., Lehmann, I., et al. 2000, AN, 321, 1

Stockdale, C. J., Romanishin, W., \& Cowan, J. J. 1998, ApJ, 508, 33

Terashima, Y., \& Wilson, A. S. 2003, ApJ, 583, 145

Trinchieri, G., Pellegrini, S., Wolter, A., Fabbiano, G., \& Fiore, F. 2000, A\&A, 364, 53

Trinchieri, G., Sulentic, J., Breitschwerdt, D., \& Pietsch, W. 2003, A\&A, 401, 173

Turner, T. J., George, I. M., Nandra, K., \& Mushotzky, R. F. 1997, ApJ, 488, 164

Turner, T. J., Perola, G. C., Fiore, F., et al. 2000, ApJ, 531, 245

Ueda, Y., Ishisaki, Y., Takahashi, T., Makashima, K., \& Ohashi, T. 2001, ApJS, 133, 1

van ben Basselaar, E. J. M., Raassen, A. J. J., Mewe, R., et al. 2003, A\&A, 411, 587

Wood, K. S., Meekins, J. F., Yentis, D. J., et al. 1984, ApJS, 56, 507 Zdziarski, A. A., Poutanen, J., \& Johnson, W. N. 2000, ApJ, 542, 703 